\title{
MILLIMETER OBSERVATIONS OF A SAMPLE OF HIGH-REDSHIFT OBSCURED QUASARS*
}

\author{
Alejo Martínez-Sansigre ${ }^{1,2}$, Alexander Karim ${ }^{1}$, Eva Schinnerer ${ }^{1}$, Alain Omont $^{3}$, Daniel J. B. Smith ${ }^{4}$, Jingwen Wu ${ }^{5}$, \\ Gary J. Hill ${ }^{6}$, Hans-Rainer KlöckneR ${ }^{2}$, Mark Lacy ${ }^{7}$, Steve Rawlings ${ }^{2}$, and Chris J. Willott ${ }^{8}$ \\ ${ }^{1}$ Max-Planck-Institut für Astronomie, Königstuhl 17, D-69117 Heidelberg, Germany; ams@ astro.ox.ac.uk, karim@mpia.de, schinner@mpia.de \\ 2 Astrophysics, Department of Physics, University of Oxford, Keble Road, Oxford OX1 3RH, UK; ams@astro.ox.ac.uk, hrk@astro.ox.ac.uk, sr@astro.ox.ac.uk \\ ${ }^{3}$ Institut d'Astrophysique de Paris, CNRS, and Université de Paris VI, 98bis boulevard Arago, F-75014 Paris, France; omont@iap.fr \\ ${ }^{4}$ The Centre for Astronomy \& Particle Theory, School of Physics and Astronomy, University of Nottingham, University Park, Nottingham NG7 2RD, UK; \\ daniel.j.b.smith@gmail.com \\ ${ }^{5}$ Harvard-Smithsonian Center for Astrophysics, 60 Garden St. MS78, Cambridge, MA 02138, USA; jwu@ cfa.harvard.edu \\ ${ }^{6}$ McDonald Observatory, University of Texas at Austin, 1 University Station C1402, Austin, TX 78712-0259, USA; hill@astro.as.utexas.edu \\ ${ }^{7}$ Spitzer Science Center, California Institute of Technology, MS220-6, 1200 E. California Boulevard, Pasadena, CA 91125, USA; mlacy@ipac.caltech.edu \\ ${ }^{8}$ Herzberg Institute of Astrophysics, National Research Council Canada, 5071 West Saanich Rd., Victoria, BC V9E 2E7, Canada; chris.willott@ nrc-cnrc.gc.ca \\ Received 2009 July 3; accepted 2009 October 7; published 2009 October 28
}

\begin{abstract}
We present observations at $1.2 \mathrm{~mm}$ with Max-Planck Millimetre Bolometer Array (MAMBO-II) of a sample of $z \gtrsim 2$ radio-intermediate obscured quasars, as well as $\mathrm{CO}$ observations of two sources with the Plateau de Bure Interferometer. The typical rms noise achieved by the MAMBO observations is $0.55 \mathrm{mJy} \mathrm{beam}^{-1}$ and five out of 21 sources $(24 \%)$ are detected at a significance of $\geqslant 3 \sigma$. Stacking all sources leads to a statistical detection of $\left\langle S_{1.2 \mathrm{~mm}}\right\rangle=0.96 \pm 0.11 \mathrm{mJy}$ and stacking only the non-detections also yields a statistical detection, with $\left\langle S_{1.2 \mathrm{~mm}}\right\rangle=0.51 \pm 0.13 \mathrm{mJy}$. At the typical redshift of the sample, $z=2,1 \mathrm{mJy}$ corresponds to a far-infrared luminosity $L_{\mathrm{FIR}} \sim 4 \times 10^{12} L_{\odot}$. If the far-infrared luminosity is powered entirely by star formation, and not by active galactic nucleus heated dust, then the characteristic inferred star formation rate is $\sim 700 M_{\odot} \mathrm{yr}^{-1}$. This far-infrared luminosity implies a dust mass of $M_{\mathrm{d}} \sim 3 \times 10^{8} M_{\odot}$, which is expected to be distributed on $\sim \mathrm{kpc}$ scales. We estimate that such large dust masses on kpc scales can plausibly cause the obscuration of the quasars. Combining our observations at $1.2 \mathrm{~mm}$ with mid- and far-infrared data, and additional observations for two objects at 350 $\mu$ m using SHARC-II, we present dust spectral energy distributions (SEDs) for our sample and derive a mean SED for our sample. This mean SED is not well fitted by clumpy torus models, unless additional extinction and far-infrared re-emission due to cool dust are included. This additional extinction can be consistently achieved by the mass of cool dust responsible for the far-infrared emission, provided the bulk of the dust is within a radius $\sim 2-3 \mathrm{kpc}$. Comparison of our sample to other samples of $z \sim 2$ quasars suggests that obscured quasars have, on average, higher far-infrared luminosities than unobscured quasars. There is a hint that the host galaxies of obscured quasars must have higher cool-dust masses and are therefore often found at an earlier evolutionary phase than those of unobscured quasars. For one source at $z=2.767$, we detect the $\mathrm{CO}(3-2)$ transition, with $S_{\mathrm{CO}} \Delta v=630 \pm 50 \mathrm{mJy} \mathrm{km} \mathrm{s}^{-1}$, corresponding to $L_{\mathrm{CO}(3-2)}=3.2 \times 10^{7} L_{\odot}$, or a brightness-temperature luminosity of $L_{\mathrm{CO}(3-2)}^{\prime}=2.4 \times 10^{10} \mathrm{~K} \mathrm{~km} \mathrm{~s}^{-1} \mathrm{pc}^{2}$. For another source at $z=4.17$, the lack of detection of the $\mathrm{CO}(4-3)$ line suggests the line to have a brightness-temperature luminosity $L_{\mathrm{CO}(4-3)}^{\prime}<1 \times 10^{10} \mathrm{~K} \mathrm{~km} \mathrm{~s}^{-1} \mathrm{pc}^{2}$. Under the assumption that in these objects the high- $J$ transitions are thermalized, we can estimate the molecular gas contents to be $M_{\mathrm{H}_{2}}=1.9 \times 10^{10} M_{\odot}$ and $<8 \times 10^{9} M_{\odot}$, respectively. The estimated gas depletion timescales are $\tau_{\mathrm{g}}=4 \mathrm{Myr}$ and $<16 \mathrm{Myr}$, and low gas-to-dust mass ratios of $M_{\mathrm{g}} / M_{\mathrm{d}}=$ 19 and $<20$ are inferred. These values are at the low end but consistent with those of other high-redshift galaxies.
\end{abstract}

Key words: galaxies: active - galaxies: high-redshift - galaxies: nuclei - galaxies: starburst - infrared: galaxies quasars: general

\section{INTRODUCTION}

Quasars are believed to be powered by supermassive black holes (SMBHs) rapidly growing by accreting matter at a large fraction of their Eddington rate. ${ }^{9}$ The accreted matter forms a disk, is heated to temperatures of $\sim 10^{3}-10^{5} \mathrm{~K}$, and emits thermal emission at optical and ultraviolet wavelengths. Clouds of gas ionized by this radiation will emit line radiation, and lines which are relatively close to the black hole have rapid orbits which will

\footnotetext{
* Based on observations carried out with the IRAM $30 \mathrm{~m}$ Telescope and the Plateau de Bure Interferometer. IRAM is supported by INSU/CNRS (France), MPG (Germany), and IGN (Spain).

9 In this article, we use the term quasar for the most powerful active galactic nuclei (AGNs). In the case of unobscured AGNs, the dividing line is taken at a $B$-band magnitude of $M_{\mathrm{B}}=-23.5$ which, assuming the typical quasar SED of Elvis et al. (1994), corresponds to $L_{\text {bol }} \gtrsim 2 \times 10^{12} L_{\odot}$. We will therefore also refer to obscured AGNs as obscured quasars if they have $L_{\text {bol }} \gtrsim 2 \times 10^{12} L_{\odot}$.
}

show broad velocity dispersions ( $>2000 \mathrm{~km} \mathrm{~s}^{-1}$ ), while clouds further away will show narrower lines.

Dust can survive when the equilibrium temperature with the ultraviolet photons is $\lesssim 2000 \mathrm{~K}$. This dust absorbs optical and ultraviolet radiation and re-emits it at infrared wavelengths characteristic of the dust temperature. If the line of sight of an observer to the central region is blocked by dust, the optical and ultraviolet continuum, as well as the broad lines, will not be observable. Such a source is known as an obscured quasar.

While radio-loud obscured quasars have been known for a long time, in the form of high-excitation narrow-line radio galaxies (see Urry \& Padovani 1995, for a review), the population of radio-quiet obscured quasars had remained elusive, with only a few individual objects known. This has changed recently, when large numbers of these sources have been found in the spectroscopic database of the Sloan Digital Sky Survey 
(Zakamska et al. 2003). Samples of spectroscopically confirmed obscured quasars have now also been identified in surveys using X-ray selection (e.g., Szokoly et al. 2004; Barger et al. 2005) as well as mid-infrared, sometimes combined with radio, selection (e.g., Houck et al. 2005; Martínez-Sansigre et al. 2005; Lacy et al. 2007). It seems that the obscured quasars are at least as common as the unobscured quasars (e.g., Reyes et al. 2008), and probably outnumber these by a $\sim 2: 1$ ratio (e.g., MartínezSansigre et al. 2005, 2008; Lacy et al. 2007; Polletta et al. 2008).

There is a large body of evidence supporting obscuration of quasars as an orientation effect, where obscured and unobscured quasars are intrinsically identical sources, only dust around the accretion disk covers certain lines of sight so that the broad emission lines and the optical and ultraviolet continuum are not visible (the dusty torus of the unified schemes, e.g., Urry \& Padovani 1995). However, dust distributed within the host galaxy can also cause obscuration, particularly if the galaxy is at an early evolutionary phase and is rich in gas and dust (see, e.g., Lawrence \& Elvis 1982; Sanders et al. 1988; Fabian 1999).

The importance of galaxy-scale dust is evident in recent studies of quasars where obscuration cannot be solely assigned to dust from a torus around the accretion disk: in some objects even the narrow lines are obscured (Martínez-Sansigre et al. 2005, 2006b; Rigby et al. 2006). The jets emanating from some obscured quasars are face-on (Martínez-Sansigre et al. 2006a; Sajina et al. 2007; Klöckner et al. 2009), something that is not expected for quasars obscured by the torus, but can be explained if the obscuring dust is on a larger $(\sim \mathrm{kpc})$ scale. The deep silicate absorption features and spectral energy distributions (SEDs) suggest foreground extinction is sometimes present (Levenson et al. 2007; Polletta et al. 2008). These all provide indirect evidence suggesting obscuring dust on $\sim \mathrm{kpc}$ scales, something which has been spectroscopically confirmed through the measurement of the $\mathrm{H} \alpha$ to $\mathrm{H} \beta$ Balmer decrement (Brand et al. 2007).

Dust present on kpc scales is expected to be relatively cool $(T \sim 50 \mathrm{~K})$ and will emit thermally in the far-infrared $(\lambda \gtrsim$ $40 \mu \mathrm{m})$, regardless of whether it is heated by young stars or by the central active galactic nucleus (AGN; see, e.g., Barvainis 1987; Sanders et al. 1989; Siebenmorgen et al. 2004; Section 3.5). Observing this dust around the peak of its emission (around rest frame $\sim 65 \mu \mathrm{m}$ ) is not optimal, since warmer dust can still contribute significantly there. The Rayleigh-Jeans tail of the emission, however, will have a much smaller contribution from warm dust, and has the additional advantage of being observable from the ground, for example, in the atmospheric windows at $850 \mu \mathrm{m}$ or $1.2 \mathrm{~mm}$.

In this paper, we present continuum observations of a sample of 21 high-redshift $(z \gtrsim 2)$ radio-intermediate obscured quasars at $1.2 \mathrm{~mm}$, combined with other infrared and submillimeter data, as well as a search for $\mathrm{CO}$ in two of the sources. The sample was selected to approximately match the break in the unobscured luminosity function at $z \sim 2\left(M_{\mathrm{B}} \sim-25.7\right.$; Croom et al. 2004), so in terms of radiation these quasars represent the energetically dominant population around the peak of the quasar activity. The sources are, however, radio-intermediate in that their radio luminosities are slightly higher than those expected from radioquiet quasars (with $L_{1.4 \mathrm{GHz}} \sim 10^{24} \mathrm{~W} \mathrm{~Hz}^{-1} \mathrm{sr}^{-1}$; MartínezSansigre et al. 2006a). Nevertheless, observations with very large baseline interferometry suggest the relative importance of the compact cores makes them more similar to the genuinely radio-quiet population than to the radio galaxies and radioloud quasars (see Klöckner et al. 2009). Section 2 summarizes the observations and data reduction. In Section 3, we show the resulting fluxes, inferred luminosities, star-formation rates (SFRs), dust masses, characteristic scale of the cool dust and discuss the possible obscuration by kpc-scale dust. Section 4 shows the results of broadband SED fitting and Section 5 presents the mean SED for our sample. In Section 6, we discuss the molecular gas observations. We compare our sample to other millimeter or submillimeter observations of $z \sim 2$ quasars in Section 7. The discussion and summary are found in Section 8.

\section{OBSERVATIONS AND DATA REDUCTION}

\subsection{MAMBO Continuum Observations}

The sample of obscured quasars was observed at a wavelength of $1.2 \mathrm{~mm}(250 \mathrm{GHz})$ using the 117-element Max-Planck Millimetre Bolometer Array (MAMBO-II; Kreysa et al. 1998, hereafter simply MAMBO), at the IRAM Pico de Veleta $30 \mathrm{~m}$ telescope. The observations were carried out in "ON-OFF" mode during the MAMBO pool at the IRAM $30 \mathrm{~m}$ telescope throughout various semesters. AMS16 was observed in winter 2005-2006, while the remaining objects were observed between summer 2007 (Sum07) and winter 2007-2008 (Win07). Due to a warning issued by IRAM (see below), the objects detected during the Win07 pool were re-observed in summer 2008 (Sum08) and winter 2008-2009 (Win08).

To minimize the effect of atmospheric absorption, the science targets were observed whenever possible at elevations $\geqslant 45^{\circ}$, and often at around $60^{\circ}$. Observing at elevations higher than $75^{\circ}$ is, however, not possible with the $30 \mathrm{~m}$ telescope.

For the first-order removal of the sky, the observations make use of a secondary mirror to chop (or "wobble"), with a typical azimuthal throw of 32 to 45 arcsec and a chopping frequency of $2 \mathrm{~Hz}$. The ON-OFF observations are done by nodding, so that the sky-only position sampled by the chopping is alternatively on the right or on the left of the sky-plus-source position. Each nod position (ON or OFF) is observed for 1 minute, and to minimize overheads an $\mathrm{ON}-\mathrm{OFF}-\mathrm{OFF}-\mathrm{ON}$ sequence is used. Hence integration blocks ("scans") are multiples of 4 minutes.

The ON-OFF observations were carried out centered on the most sensitive pixel, number 20, with the atmospheric opacity $\tau_{230 \mathrm{GHz}} \lesssim 0.3$ and medium-to-low sky noise $(\lesssim 200 \mathrm{mJy}$ beam $\left.^{-1}\right)$. Each source was typically observed in several blocks of 20 minute integrations.

The "sky-only" position can sometimes fall on a real astronomical source. Given that high-redshift AGNs are often found to have (sub)millimeter-bright galaxies nearby (Stevens et al. 2003), there is a real risk of ruining the sky subtraction by chopping onto a bright source. To minimize the risk of this happening (and the impact on the final data), the chop angle ("wobbler throw") was alternatively varied between 32,35 , and 42 arcsec, and observations were carried out on different dates and at different times of the day. This means the chop in azimuth corresponds to different physical offsets in R.A. and decl., so the chance of repeatedly falling on the same astronomical source is minimized.

Throughout the observing runs, gain calibration was performed by observing Neptune, Uranus or Mars, and the flux calibration was always found to be accurate to $\leqslant 20 \%$. The gain calibration was also monitored regularly by observing other sources with known bright millimeter fluxes $(\geqslant 5 \mathrm{Jy})$. Total power measurements at different elevations were made to infer the atmospheric opacity ("skydip"). These were performed 
Table 1

Observed Properties of the Sample of Obscured Quasars

\begin{tabular}{|c|c|c|c|c|c|c|c|c|}
\hline Name & $\begin{array}{c}\text { R.A. } \\
(\mathrm{J} 2000)\end{array}$ & Decl. & $z_{\text {spec }^{a}}$ & Optical $^{\text {b }}$ & Radio $^{c}$ & $f_{\text {core }} \mathrm{d}^{\mathrm{d}}$ & Mid-Infrared & $\begin{array}{l}S_{1.2 \mathrm{~mm}}^{\mathrm{f}} \\
(\mathrm{mJy})\end{array}$ \\
\hline AMS01 & 171311.17 & +595551.5 & $\cdots$ & B & SS & $<0.3$ & $\mathrm{C}$ & $0.63 \pm 0.53$ \\
\hline AMS03 & 171340.19 & +592745.8 & 2.698 & NL & SS & 0.8 & $\ldots$ & $0.76 \pm 0.51$ \\
\hline AMS04 & 171340.62 & +594917.1 & 1.782 & NL & SS & $\cdots$ & $\mathrm{S}$ & $0.55 \pm 0.58$ \\
\hline AMS05 & 171342.77 & +593920.2 & 2.850 & NL & GP & 1.1 & $\mathrm{~S}$ & $-0.26 \pm 0.40$ \\
\hline AMS07 & 171402.25 & +594828.8 & $\cdots$ & B & FS & $\cdots$ & $\mathrm{C}$ & $1.05 \pm 0.44$ \\
\hline AMS08 & 171429.67 & +593233.5 & 1.979 & NL & $\cdots$ & $\cdots$ & $\mathrm{S}$ & $1.99 \pm 0.34$ \\
\hline AMS09 & 171434.87 & +585646.4 & 2.1 & $\mathrm{~B}$ & SS & 0.9 & $\mathrm{~S}$ & $0.51 \pm 0.56$ \\
\hline AMS10 & 171620.08 & +594026.5 & $\cdots$ & B & SS & $\cdots$ & $\mathrm{C}$ & $0.62 \pm 0.79$ \\
\hline AMS11 & 171821.33 & +594027.1 & 1.6 & B & SS & $\ldots$ & $\mathrm{P}, \mathrm{S}$ & $0.36 \pm 0.57$ \\
\hline AMS16 & 171942.07 & +584708.9 & 4.169 & NL & GP & $<0.6$ & $\cdots$ & $2.12 \pm 0.54$ \\
\hline AMS17 & 172045.17 & +585221.3 & 3.137 & NL & SS & $<0.3$ & $\mathrm{P}, \mathrm{S}$ & $1.73 \pm 0.48^{h}$ \\
\hline AMS18 & 172046.32 & +6002 29.6 & 1.6 & B & FS & $\cdots$ & $\mathrm{S}$ & $1.11 \pm 0.53$ \\
\hline AMS19 & 172048.00 & +594320.7 & 2.3 & B & FS & 1.0 & $\mathrm{~S}$ & $2.76 \pm 0.66$ \\
\hline AMS20 & 172059.10 & +591750.5 & $\ldots$ & B & GP & $\ldots$ & $\mathrm{C}$ & $-0.78 \pm 0.93$ \\
\hline AMS21 & 172120.09 & +590348.6 & 1.8 & B & SS & 0.7 & $\mathrm{P}, \mathrm{S}$ & $0.52 \pm 0.65$ \\
\hline
\end{tabular}

Notes.

${ }^{a}$ Redshifts with three decimal places are from optical spectroscopy (Martínez-Sansigre et al. 2006b), while those with only one decimal place are from mid-infrared spectroscopy (Martínez-Sansigre et al. 2008). The redshift for AMS05 is from the optical spectrum taken by Smith et al. (2009).

b Summary of optical spectroscopy properties. B stands for blank spectrum, NL for narrow lines.

c Summary of radio spectral properties from Martínez-Sansigre et al. (2006a). SS stands for steep spectrum, FS for flat spectrum, and GP for gigahertz-peaked source.

d Approximate fraction of radio flux at $1.4 \mathrm{GHz}$ recovered at VLBI resolution at $1.6 \mathrm{GHz}$ (Klöckner et al. 2009).

e Spectral properties in the mid-infrared (see Martínez-Sansigre et al. 2008). C stands for continuum only, S for silicate absorption,

$\mathrm{P}$ for polycyclic aromatic hydrocarbons (PAHs).

${ }^{\mathrm{f}}$ Flux densities at $1.2 \mathrm{~mm}$. Significant detections are marked in bold.

g Flux density for AMS13 taken from Lutz et al. (2005), their source 22204.

${ }^{\mathrm{h}}$ Flux density for AMS17 taken from Sajina et al. (2008), their source 22558.

typically every $2 \mathrm{hr}$ under stable weather conditions, and at shorter intervals for less stable conditions. The skydips were generally done with the telescope azimuthal angle matched to that of the science targets. The focus of the telescope was also regularly monitored on bright sources. Before the first science target and every 40 minutes the accuracy of the telescope pointing was checked using the nearby source J1638 + 573 (0.6 Jy). Observations of $\mathrm{J} 1638+573$ and other bright sources were also used to check for anomalous refraction, in which case no science observations were made.

During the observations, the sensitivity of MAMBO in a $1 \mathrm{~s}$ integration was typically between 35 and $45 \mathrm{mJy}_{\text {beam }}^{-1}$. Including typically $60 \%$ overheads, the observations of this sample of 21 obscured quasars totalled approximately $65 \mathrm{hr}$.

The data were reduced using the MOPSIC pipeline (developed by R. Zylka). MOPSIC removes the atmospheric emission using the two chopper positions. ${ }^{10}$ The sky noise is measured by taking the weighted mean of the correlated signal from adjacent pixels, and subsequently removed. The MAMBO pixels are separated in the array by two beams and weak point sources $(<150 \mathrm{mJy})$ will therefore not be detected in adjacent pixels. Using the measurements of the atmospheric opacity from the skydips, and the elevation at which objects were observed, the

\footnotetext{
${ }^{10}$ See http://iram.fr/IRAMFR/ARN/dec05/node9.html for more details.
}

extinction due to atmospheric water vapor is then corrected. Gain calibration converts counts to flux density, and finally, for each science target the weighted mean of all the scans is taken. These are the flux densities quoted in Table 1: a relatively uniform root mean square (rms) noise $\sim 0.55 \mathrm{mJy} \mathrm{beam}^{-1}$ was achieved for most of the sources, with only a few sources having an rms $\sim 0.7-0.9$ mJy beam $^{-1}$.

In 2008 September, IRAM issued a warning to MAMBO observers: several technical problems had occurred during the Win07 semester, which included thermal and electric malfunctions in the preamplifier, heightened sensitivity of the bolometer to microphotonics and spikes in the detected signal. These problems were expected to affect a fraction of the scans, and the warning suggested that any $<10 \mathrm{mJy}$ detections obtained during this semester should be checked. Four of sources from this sample were detected during Win07: AMS07, AMS08 AMS12, and AMS17. All four were observed again during the Win08 period, together with extra scans of AMS10, AMS11, and AMS20. The data for Win07 and Win08 were reduced independently, with the results shown in Table 2 .

The Win07 flux densities are systematically higher, so only sources detected in the Sum08/Win08 period are considered safe detections. However, the source AMS17 was independently detected by Sajina et al. (2008, their source 22558), so their flux density is used in Table 1. The source AMS07 is not considered 
Table 2

Comparison of Resulting Fluxes in Win07 and Sum08/Win08

\begin{tabular}{lccc}
\hline \hline Name & $\begin{array}{c}\text { Win07 } \\
(\mathrm{mJy})\end{array}$ & $\begin{array}{c}\text { Sum08/Win08 } \\
(\mathrm{mJy})\end{array}$ & $\begin{array}{c}\text { Combined } \\
(\mathrm{mJy})\end{array}$ \\
\hline AMS07 & $2.43 \pm 0.83$ & $\mathbf{1 . 0 5} \pm \mathbf{0 . 4 4}$ & $1.27 \pm 0.41$ \\
AMS08 & $2.04 \pm 0.59$ & $\mathbf{1 . 9 9} \pm \mathbf{0 . 4 1}$ & $1.99 \pm 0.34$ \\
AMS10 & $0.81 \pm 0.93$ & $0.45 \pm 1.1$ & $\mathbf{0 . 6 2} \pm \mathbf{0 . 7 9}$ \\
AMS11 & $0.83 \pm 0.64$ & $-0.59 \pm 1.00$ & $\mathbf{0 . 3 6} \pm \mathbf{0 . 5 7}$ \\
AMS12 & $4.03 \pm 1.03$ & $\mathbf{3 . 7 4} \pm \mathbf{0 . 6 0}$ & $3.80 \pm 0.55$ \\
AMS17 & $2.39 \pm 0.55$ & $1.45 \pm 0.62$ & $1.94 \pm 0.44$ \\
AMS20 & $-0.16 \pm 1.08$ & $-1.24 \pm 1.56$ & $-\mathbf{0 . 7 8} \pm \mathbf{0 . 9 3}$ \\
\hline
\end{tabular}

Notes. Bold shows the values used in Table 1.

a The flux density for AMS17 used in Table 1, $1.73 \pm 0.48$, is taken from Sajina et al. (2008), their source 22558.

a safe detection. The potential worry was detecting sources spuriously so for the sources that are clearly not detected the combined data can be used to yield deeper limits. Table 2 marks in bold the flux densities that are used in Table 1.

\subsection{SHARC Observations}

Two objects were observed in continuum at $350 \mu \mathrm{m}$, AMS13 and AMS16, on 2005 June 16 and 17, using the Submillimeter High Angular Resolution Camera II (SHARC-II) at the $10.4 \mathrm{~m}$ telescope of the Caltech Submillimeter Observatory (CSO) at Mauna Kea, Hawaii (Dowell et al. 2003). SHARC-II is a background-limited camera utilizing a "CCD-style" bolometer array with $12 \times 32$ pixels. At $350 \mu \mathrm{m}$, the beam size is 8.5 arcsec, with a $2.59 \times 0.97 \mathrm{arcmin}^{2}$ field of view. The observations were taken during very dry weather: AMS16 was observed during $2 \mathrm{hr}$ with an opacity at $225 \mathrm{GHz}$ of $\tau_{225 \mathrm{GHz}}=0.05$, and AMS13 was observed for $1 \mathrm{hr}$ with $\tau_{225 \mathrm{GHz}}=0.06$. The Dish Surface Optimization System (DSOS; Leong et al. 2006) was used during the observation to correct the dish surface figure for imperfections and gravitational deformations as the dish moves in elevation.

The raw data were reduced with the Comprehensive Reduction Utility for SHARC-II, CRUSH-version 1.40a9-2 (Kovács 2006). We used the sweep mode of SHARC-II to observe the sources: in this mode the telescope moves in a Lissajous pattern that keeps the central regions of the maps fully sampled, but causes the edges to be much noisier than the central regions. Pointing was checked with Uranus and secondary object CRL_16293-2422 several times during the observations. The pointing accuracy is better than 3 arcsec. Uranus was also used as the flux calibrator. We used Starlink's "stats" package to measure the flux of the calibrator and estimate the noise of the image. The resulting rms noise values for AMS13 and AMS16 are $7.0 \mathrm{mJy} \mathrm{beam}^{-1}$ and $5.5 \mathrm{mJy}^{\text {beam }}{ }^{-1}$, respectively, and neither source is detected at the $3 \sigma$ level.

\subsection{PdBI Observations}

Two sources, AMS12 and AMS16, were observed using the Plateau de Bure Interferometer (PdBI) to search for molecular gas in the host galaxies, by using carbon monoxide (CO) as a tracer of the molecular gas.

AMS16 was the first object observed with MAMBO in 2005. At the redshift of the source, $z=4.169$, the $\mathrm{CO}(4-3)$ rotational transition falls in the $3 \mathrm{~mm}$ atmospheric window (where (4-3) stands for the transition from the $J=4$ to the $J=3$ angular quantum numbers).
The observations were carried out on 2008 August 28 and 2007 September 1, with five antennae in the most compact configuration (D). The source 3C 345 was used as amplitude and bandpass calibrator, MWC 349 as amplitude calibrator, and the phase calibrators were $1823+568$ (28 Aug.) and $1637+574$ (01 Sep.). The correlator was configured with both polarizations observing at a central frequency of $89.2002 \mathrm{GHz}$, with a bandwidth of $1 \mathrm{GHz}$. For the $\mathrm{CO}$ (4-3) transition at $z=4.169$, this corresponds to $\pm 1680 \mathrm{~km} \mathrm{~s}^{-1}$, and should suffice to avoid missing the line given the accuracy of the redshift.

The night of August 28 had moderately good conditions, and $25 \%$ of the data were flagged automatically. ${ }^{11}$ The night of September 1 had excellent conditions (with only $1 \%$ of the data flagged) although for one antenna, 1 spectral unit out of the 8 (L07 in horizontal polarization for antenna 6) had to be flagged for the entire night. The total usable time from both nights amounted to $12.9 \mathrm{hr}$ on-source.

The data were reduced using the GILDAS software. ${ }^{12}$ After amplitude, phase, and bandpass calibration, data cubes of the phase calibrators and of AMS16 were created. The phase calibrators came out perfectly centered at the phase center, suggesting excellent phase calibration, but AMS16 was not detected either in continuum or in line. A spectrum was extracted at the position of the source, with the shape of the beam. The rms noise achieved in the spectrum was $0.65 \mathrm{mJy} \mathrm{beam}^{-1}$ per $10 \mathrm{MHz}$ bin. Assuming a box-car CO line shape with full width to a zero intensity of $400 \mathrm{~km} \mathrm{~s}^{-1}$, this corresponds to $74 \mathrm{mJy}$ beam $^{-1} \mathrm{~km} \mathrm{~s}^{-1}$. The continuum sensitivity achieved over the entire bandwidth was $0.065 \mathrm{mJy}^{\text {beam }}{ }^{-1} \mathrm{rms}$.

AMS12 was also observed using the PdBI, to look for the CO (3-2) transition. The observations, carried out during the 2009 April 30 and May 13, were centered on $91.796 \mathrm{GHz}$ using a $1 \mathrm{GHz}$ bandwidth. Depending on the day, the calibrators 3C 345, MWC 349, $2145+067$, and $0923+392$ were used as flux or bandpass calibrators, while the source $1637+574$ was used as a phase calibrator on both dates. On April 30, only five antennae were available and in addition approximately $50 \%$ of the data were flagged. For both dates the water vapor radiometer for antenna 2 did not work, so no atmospheric phase corrections were applied to the data from this antenna. In total, $7.3 \mathrm{hr}$ of usable data were obtained and an rms noise of $0.70 \mathrm{mJy}^{\text {beam }}{ }^{-1}$ per $10 \mathrm{MHz}$ channel was reached.

The CO (3-2) transition was clearly detected in AMS12, with an integrated flux of $630 \mathrm{mJy}$ beam $^{-1} \mathrm{~km} \mathrm{~s}^{-1}$. The continuum rms is $0.1 \mathrm{mJy}^{\text {beam }}{ }^{-1}$, and no continuum is detected at $3 \mathrm{~mm}$, so that we obtain a $3 \sigma$ limit of $S_{3 \mathrm{~mm}}<0.3 \mathrm{mJy}$. In the case of AMS16, the lack of continuum sets an $3 \sigma$ upper limit on the $89 \mathrm{GHz}(3 \mathrm{~mm})$ flux density of $S_{3 \mathrm{~mm}}<0.2 \mathrm{mJy}$.

\section{RESULTS FROM CONTINUUM OBSERVATIONS}

The millimeter continuum observations provide us with a wealth of information about the far-infrared luminosities and cool-dust masses of our sources. They can also be used to test whether there is enough dust along the host galaxy to cause the obscuration in the quasars. In the following section, we derive these physical properties from our observations.

\footnotetext{
11 Data can be flagged as having bad quality for many reasons. Examples for reasons to automatically flag the data during the observations are time errors, tracking problems, or antenna shadowing. During the calibration and reduction of the data, visibilities with high phase noise or high pointing correction, tracking errors as well as amplitude loss can also be flagged.

12 http://www.iram.fr/IRAMFR/GILDAS
} 


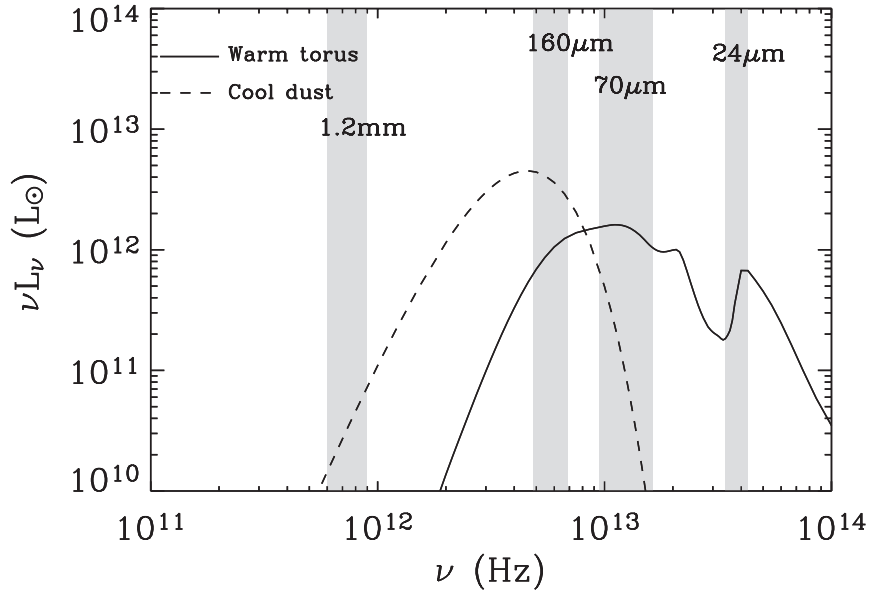

Figure 1. Illustration of the physical components that dominate at each observed band. The figure shows the SED of a model torus (solid line, from Nenkova et al. 2008a, 2008b) and a cool-dust component (dashed line) modeled as a gray body with temperature $T=40 \mathrm{~K}$ and emissivity index $\beta=1.5$ (see Equation (2)). The observed bands at 24,70, and $160 \mu \mathrm{m}$, as well as $1.2 \mathrm{~mm}$, are available for all 21 sources and have been marked in gray. This model source is assumed to be at $z=2$ so the bands have been accordingly shifted to the rest-frame frequencies. The relative luminosities of the two components are arbitrary but representative of the sources in this sample. For completeness, the exact torus parameters used are: single-cloud optical depths $\tau_{\mathrm{V}}=60$, Gaussian angular distribution of clumps around the equator, $\sigma=60$, the number of clouds along a ray in the equatorial plane $N=10$, the ratio of inner to outer torus radius $Y=30$, and the power of the radial density distribution, $q=0$.

The data uniformly available for this sample include the following wavelengths: 3.6, 4.5, 5.8, 8.0, 24, 70, and $160 \mu \mathrm{m}$, as well as $1.2 \mathrm{~mm}$. For a small number of sources, data are also available at 350 or $850 \mu \mathrm{m}$. With such excellent wavelength coverage, one might expect the far-infrared luminosities and cool-dust masses to be very accurately constrained. However, the wavelengths corresponding to the rest-frame mid-infrared are dominated by hotter dust from the warm torus around the central engine, and cannot be used to constrain the cooldust component as we explain below (this was originally by selection, but has additionally been confirmed by mid-infrared spectroscopy, see Martínez-Sansigre et al. 2006b, 2008).

The far-infrared luminosity is dominated by cool dust, expected to be characterized by temperatures $\sim 35-50 \mathrm{~K}$. This means that emission is expected to peak around 65-90 $\mu \mathrm{m}$ and drop exponentially at shorter wavelengths. The emission from the warm torus is caused by dust at a range of temperatures, typically $\sim 100-2000 \mathrm{~K}$, whose emission will peak at shorter wavelengths than that of the cool dust. If the AGN is powerful enough, as is the case in the sources making up this sample, the dust emission from the torus will dominate at mid-infrared wavelengths. Hence, the mid-infrared emission is not useful in constraining the cool-dust mass and luminosity.

To illustrate this, Figure 1 shows a model rest-frame SED consisting of cool dust modeled by a gray body with temperature $T=40 \mathrm{~K}$ and emissivity $\beta=1.5$ together with a model AGN torus from the radiative transfer models of Nenkova et al. (2008a, 2008b). The Spitzer MIPS bands at 24, 70, and $160 \mu \mathrm{m}$, as well as the MAMBO band at $1.2 \mathrm{~mm}$ have been marked in gray, assuming this source to be at $z=2$. The emission seen at $1.2 \mathrm{~mm}$ will be completely dominated by the cool dust, while the emission at $24 \mu \mathrm{m}$ will be completely dominated by the warm dust of the torus. The $70 \mu \mathrm{m}$ band is expected to be dominated by the torus, with some contribution from the cooler dust, while the situation for the emission observed at $160 \mu \mathrm{m}$ is expected

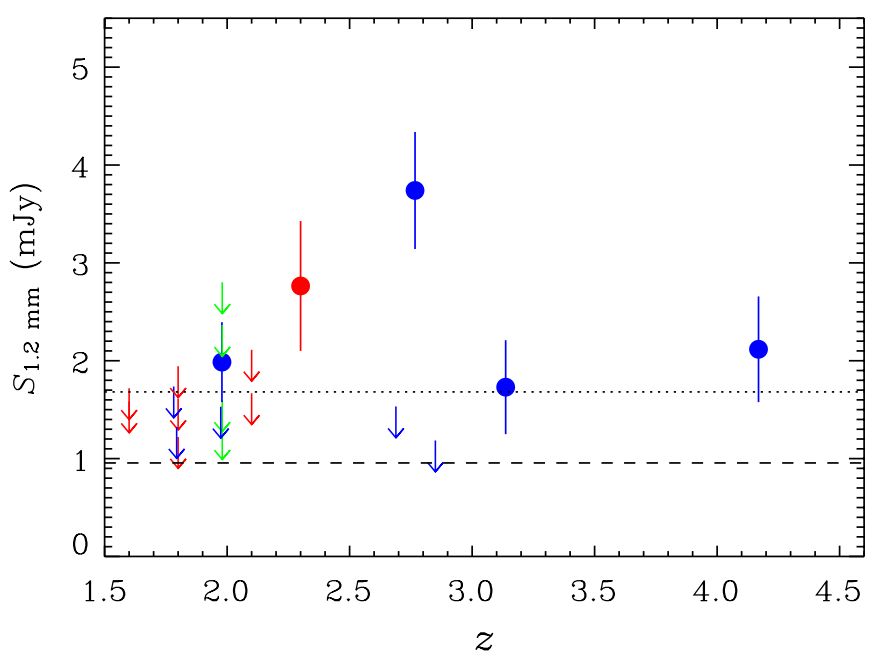

Figure 2. Flux density, $S_{1.2} \mathrm{~mm}$, vs. redshift $z$ for the sample of obscured quasars. Sources marked in blue have redshifts from optical spectroscopy, those marked in red have redshifts only from their mid-infrared spectra. All limits are $3 \sigma$. The four green sources have no spectroscopic redshifts and have been placed at $z=1.98$, the median value for sources with a spectroscopic redshift. At $z=2$ there are four green sources (one detection and three upper limits) and two blue sources (one detection and one upper limit). The dotted line represents the approximate flux density limit of the MAMBO observations: $\sim 1.65 \mathrm{mJy}$. The dashed line shows the value of $\left\langle S_{1.2} \mathrm{~mm}\right\rangle$, and the mean flux density for all 21 sources.

to be reverse: the cool dust dominates with some contribution from the torus.

This is only an illustration, and the relative importance of each component will vary depending on the actual temperature of the cool dust, the actual SED of the torus and their relative luminosities. However, Figure 1 does show that when a powerful AGN is present, the data at 24 and $70 \mu \mathrm{m}$ and the mid-infrared spectra will not provide very powerful constraints on the cooldust emission. These are the data at $1.2 \mathrm{~mm}$ that will provide the best measurement of this emission. The data at $160 \mu \mathrm{m}$ should provide useful constraints but for most sources in our sample they are too shallow to provide detections. Data at 350 or $850 \mu \mathrm{m}$ are extremely useful but are only available for three sources.

Hence, we will determine the far-infrared luminosities based on the flux densities at $1.2 \mathrm{~mm}$ alone for most of the sources in this sample. For sources where the data at 160, 350, or $850 \mu \mathrm{m}$ provide a useful constrain, we will make use of these data too. However, the $24 \mu \mathrm{m}$ data will not be used to constrain the cooldust emission (and in most cases, neither will the $70 \mu \mathrm{m}$ data).

\subsection{Flux Densities at $1.2 \mathrm{~mm}$}

The MAMBO observations yield detections at the $\geqslant 3 \sigma$ level for five out of 21 sources observed (24\%). The results are summarized in Table 1 and Figure 2 (where sources with no spectroscopic redshift were placed at $z=1.98$, the median for sources with a spectroscopic redshift). The mean noise achieved is $0.56 \mathrm{mJy}^{\text {beam }}{ }^{-1}$, the median is $0.54 \mathrm{mJy}_{\text {beam }}{ }^{-1}$. Hence, for the non-detections, typical $3 \sigma$ limits $\sim 1.65 \mathrm{mJy}$ are obtained.

With $76 \%$ of the sources undetected, the median flux density does not provide a useful characteristic number for the sample. A more meaningful number is the mean flux density at $1.2 \mathrm{~mm}$ for the entire sample. The flux densities of all sources were 


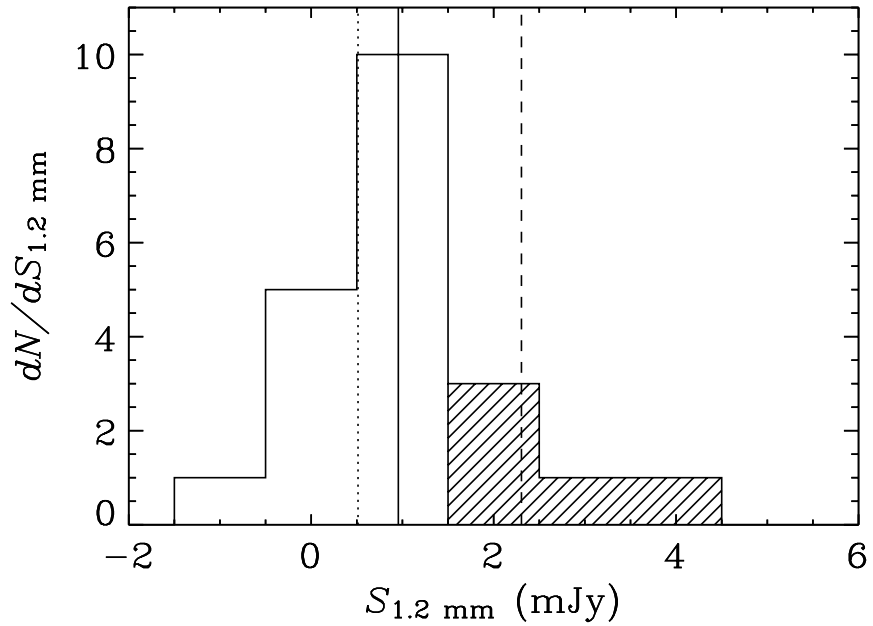

Figure 3. Distribution of observed flux densities. The empty histogram represents the measured flux densities for all sources, the shaded histogram for detections. The distribution is clearly centered on a positive value, and skewed toward the high-flux density end. The solid vertical line shows the stacked mean of all sources, the dashed line the stacked mean for detections, and the dotted line the stacked mean for non-detections.

therefore combined using a weighted mean:

$$
\left\langle S_{1.2 \mathrm{~mm}}\right\rangle=\frac{\left(\sum_{i} w_{i} S_{1.2 \mathrm{~mm} i}\right)}{\left(\sum_{i} w_{i}\right)} \pm\left(\sum_{i} w_{i}\right)^{-1 / 2}
$$

where $w_{i}=1 / \sigma_{i}^{2}$. This yielded a statistical detection, with $\left\langle S_{1.2 \mathrm{~mm}}\right\rangle=0.96 \pm 0.11 \mathrm{mJy}$. A potential worry is that a few bright sources might be causing the statistical detection, while the rest of the sources are intrinsically faint. Stacking only the non-detections still yields a detection at the $3.9 \sigma$ level: $\left\langle S_{1.2 \mathrm{~mm}}\right\rangle=0.51 \pm 0.13 \mathrm{mJy}$. Stacking all the detections leads to a mean flux density of $\left\langle S_{1.2 \mathrm{~mm}}\right\rangle=2.30 \pm 0.23 \mathrm{mJy}$. Figure 3 shows the distribution of flux densities (from Table 1), which is centered on a positive value and skewed toward the higher flux densities. This figure supports the statement that the typical flux density of an object from this sample is $\sim 0.5-1.0 \mathrm{mJy}$. If the actual flux densities of the sources were so low that the distribution were dominated by the noise, Figure 3 would be centered on 0 , with a symmetric characteristic width $\sim 0.55 \mathrm{mJy}$, and only a slight skew toward the high fluxes (the detections with $S_{v} \gtrsim 1.65 \mathrm{mJy}$ ).

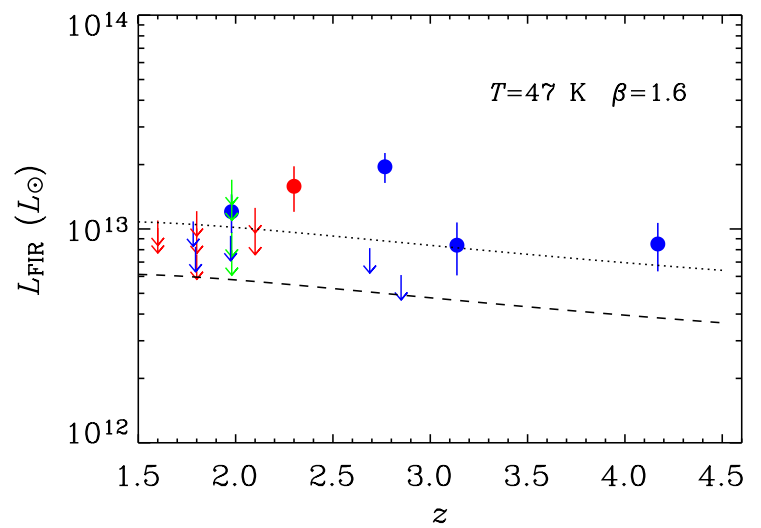

We now consider whether the detection at $1.2 \mathrm{~mm}$ is related to the presence or lack of narrow lines in the optical spectra. Of the 5 detections at $1.2 \mathrm{~mm}$, four show narrow lines $(80 \%)$ and only one $(20 \%)$ has a blank optical spectrum (AMS19). Alternatively, of the nine objects with narrow lines, four have detections at $1.2 \mathrm{~mm}(44 \%)$, while of the 12 with no narrow lines, only one is detected ( $8 \%)$. Due to the small numbers, no trend is significant, but at first sight the data hint that sources with narrow lines are more likely to be detected at $1.2 \mathrm{~mm}$.

Looking at the stacked flux densities for narrow-line and for blank objects (no narrow lines) suggests the dust content to be similar: the weighted mean for narrow-line sources is $1.09 \pm$ $0.16 \mathrm{mJy}$, while for blank sources it is $0.81 \pm 0.16 \mathrm{mJy}$, so both are statistically detected. Although at first sight the difference appears significant, the narrow-line objects include AMS12 with a flux of $3.7 \mathrm{mJy}$ which is affecting the mean: the mean of the other eight narrow-line objects excluding AMS12 is $0.76 \mathrm{mJy}$, very similar to that of the objects with no narrow lines. Hence, the mean mm flux densities for both narrow-line and blank objects appear similar.

\subsection{Far-infrared Luminosities}

Given a flux density, the infrared luminosity of a gray body is given by

$$
\begin{aligned}
& L_{\mathrm{FIR}}= \\
& \frac{15}{\pi^{5}} \frac{4 \pi d_{\mathrm{L}}^{2}}{(1+z)}\left(\frac{k_{\mathrm{B}}}{h_{\mathrm{P}} v_{\mathrm{rf}}}\right)^{\beta} \frac{\Gamma(4+\beta) \zeta(4+\beta) \sigma_{\mathrm{SB}} T^{4+\beta} S_{1.2 \mathrm{~mm}}}{B_{\nu_{\mathrm{rf}}}\left(T, v_{\mathrm{rf}}\right)}
\end{aligned}
$$

where $h_{\mathrm{P}}$ is Planck's constant, $k_{\mathrm{B}}$ is Boltzmann's constant, $\sigma_{\mathrm{SB}}$ is the Stefan-Boltzmann constant, and $d_{\mathrm{L}}$ is the luminosity distance at redshift $z$. The terms $v$ and $v_{\mathrm{rf}}$ refer to observed and rest-frame frequency respectively, $v_{\mathrm{rf}}=(1+z) \nu$. The term $B_{v_{\mathrm{rf}}}\left(T, v_{\mathrm{rf}}\right)$ is the Planck radiation function:

$$
B_{v_{\mathrm{rf}}}\left(T, v_{\mathrm{rf}}\right) \equiv \frac{2 h_{P} v_{\mathrm{rf}}^{3}}{c^{2}} \frac{1}{\left(e^{\frac{h_{\mathrm{p}} v_{\mathrm{rf}}}{k_{\mathrm{B}} T}}-1\right)} .
$$

Figure 4 and Table 3 show the inferred values of $L_{\mathrm{FIR}}$ assuming two different gray bodies, one with $T=47 \mathrm{~K}$ and $\beta=1.6$ (representative of unobscured quasars at high redshift, see Beelen et al. 2006), the other with $T=35 \mathrm{~K}$ and $\beta=1.5$ (representative of submillimeter galaxies, SMGs; see Kovács et al. 2006). The value of $\left\langle S_{1.2 \mathrm{~mm}}\right\rangle$, also assuming $z=1.98$,

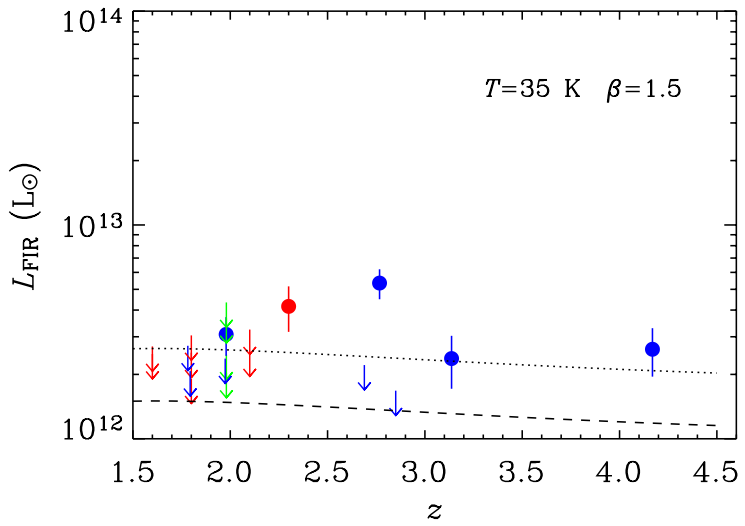

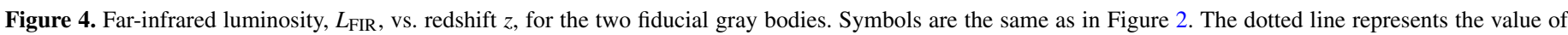

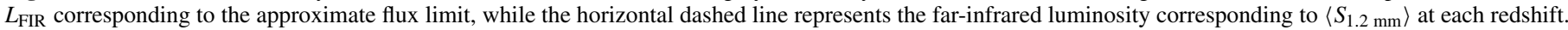


Table 3

Inferred Properties of the Sample of Obscured Quasars

\begin{tabular}{|c|c|c|c|c|c|c|c|c|}
\hline \multirow[t]{2}{*}{ Name } & \multirow[t]{2}{*}{$\log _{10}\left[L_{\mathrm{bol}} / L_{\odot}\right]^{\mathrm{a}}$} & \multirow[t]{2}{*}{$A_{\mathrm{V}}{ }^{\mathrm{a}}$} & \multicolumn{3}{|c|}{$T=47 \mathrm{~K}, \beta=1.6^{\mathrm{b}}$} & \multicolumn{3}{|c|}{$T=35 \mathrm{~K}, \beta=1.5^{\mathrm{c}}$} \\
\hline & & & $\log _{10}\left[L_{\mathrm{FIR}} / L_{\odot}\right]$ & $\log _{10}\left[L_{\mathrm{FIR}} / L_{\odot}\right]$ & $\log _{10}\left[\mathrm{SFR} / M_{\odot} \mathrm{yr}^{-1}\right]$ & $\log _{10}\left[M_{\mathrm{d}} / M_{\odot}\right]$ & $\log _{10}\left[\mathrm{SFR} / M_{\odot} \mathrm{yr}^{-1}\right]$ & $\log _{10}\left[M_{\mathrm{d}} / M_{\odot}\right]$ \\
\hline AMS01 & $\geqslant 13.2$ & $\geqslant 40.5$ & $<13.0$ & $<3.2$ & $<8.7$ & $<12.4$ & $<2.6$ & $<8.9$ \\
\hline AMS02 & 12.4 & 0.5 & $<13.0$ & $<3.2$ & $<8.7$ & $<12.4$ & $<2.6$ & $<8.9$ \\
\hline AMS03 & $\geqslant 13.2$ & $\geqslant 21.5$ & $<12.9$ & $<3.2$ & $<8.6$ & $<12.3$ & $<2.6$ & $<8.9$ \\
\hline AMS04 & 13.7 & 68.5 & $<13.0$ & $<3.3$ & $<8.7$ & $<12.4$ & $<2.7$ & $<9.0$ \\
\hline AMS05 & 13.6 & $5.5^{\mathrm{d}}$ & $<12.8$ & $<3.0$ & $<8.5$ & $<12.2$ & $<2.5$ & $<8.8$ \\
\hline AMS06 & 13.1 & 12.0 & $<12.9$ & $<3.1$ & $<8.6$ & $<12.3$ & $<2.5$ & $<8.8$ \\
\hline AMS07 & $\geqslant 12.9$ & $\geqslant 17.0$ & $<12.9$ & $<3.1$ & $<8.6$ & $<12.3$ & $<2.5$ & $<8.8$ \\
\hline AMS08 & $\geqslant 13.6$ & $\geqslant 65.0$ & 13.1 & 3.3 & 8.8 & 12.5 & 2.7 & 9.0 \\
\hline AMS09 & 13.1 & 16.0 & $<13.0$ & $<3.2$ & $<8.7$ & $<12.4$ & $<2.6$ & $<8.9$ \\
\hline AMS10 & $\geqslant 13.1$ & $\geqslant 55.0$ & $<13.2$ & $<3.4$ & $<8.9$ & $<12.6$ & $<2.8$ & $<9.1$ \\
\hline AMS11 & 12.7 & 13.0 & $<13.0$ & $<3.3$ & $<8.7$ & $<12.4$ & $<2.7$ & $<9.0$ \\
\hline AMS12 & 13.3 & 21.0 & 13.3 & 3.5 & 9.0 & 12.7 & 3.0 & 9.3 \\
\hline AMS13 & 13.9 & 22.5 & $<13.0$ & $<3.2$ & $<8.7$ & $<12.4$ & $<2.6$ & $<8.9$ \\
\hline AMS14 & $\geqslant 13.8$ & $\geqslant 73.5$ & $<12.9$ & $<3.2$ & $<8.6$ & $<12.3$ & $<2.6$ & $<8.9$ \\
\hline AMS15 & $\geqslant 13.0$ & $\geqslant 40.0$ & $<13.1$ & $<3.3$ & $<8.8$ & $<12.5$ & $<2.8$ & $<9.1$ \\
\hline AMS16 & 13.9 & 21.0 & 12.9 & 3.2 & 8.6 & 12.4 & 2.7 & 9.0 \\
\hline AMS17 & 13.8 & 33.5 & 12.9 & 3.2 & 8.6 & 12.4 & 2.6 & 8.9 \\
\hline AMS18 & $\geqslant 14.2$ & $\geqslant 100.0$ & $<13.0$ & $<3.2$ & $<8.7$ & $<12.4$ & $<2.6$ & $<8.9$ \\
\hline AMS19 & 13.4 & 7.5 & 13.2 & 3.4 & 8.9 & 12.6 & 2.9 & 9.2 \\
\hline AMS20 & 12.7 & 0.2 & $<13.2$ & $<3.5$ & $<8.9$ & $<12.6$ & $<2.9$ & $<9.2$ \\
\hline AMS21 & 13.1 & 24.0 & $<13.1$ & $<3.3$ & $<8.8$ & $<12.5$ & $<2.7$ & $<9.0$ \\
\hline
\end{tabular}

Notes.

${ }^{a}$ Bolometric luminosities and extinctions derived from the broadband data between 3.6 and $24 \mu \mathrm{m}$, as described in Section 4 .

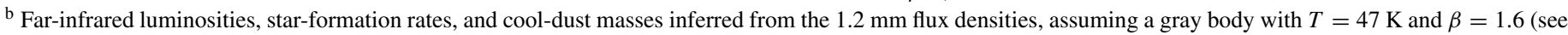
Section 3).

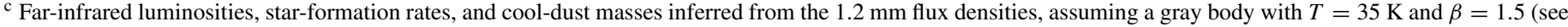
Section 3).

d The value of $A_{\mathrm{V}}=5.5$ was inferred by Smith et al. (2009) using additional data at 1.2, 1.7, and $2.2 \mu \mathrm{m}$.

corresponds to $\left\langle L_{\mathrm{FIR}}\right\rangle=6.3 \pm 0.7 \times 10^{12} L_{\odot}(T=47 \mathrm{~K}$ and $\beta=1.6$ ) or $1.6 \pm 0.2 \times 10^{12} L_{\odot}$ (for $T=35 \mathrm{~K}$ and $\beta=$ $1.5)$. Hence, the obscured quasars are typically ultra-luminous in the far-infrared, with a characteristic luminosity of $\sim 4 \times$ $10^{12} L_{\odot}$ (the mean of both values).

Despite the range in spectroscopic redshifts $(1.6 \leqslant z \leqslant 4.2)$, the relatively flat selection function at $1.2 \mathrm{~mm}$ means that the detection of sources is primarily determined by their far-infrared luminosities, and is only weakly dependent on redshift (see Figure 4). In fact, for a given flux density, the corresponding farinfrared luminosity is actually smaller at high redshift. Hence intrinsically less luminous objects are easier to detect at higher redshift. This probably explains why in our sample, the higher redshift sources $(z \gtrsim 2)$ have a slightly higher detection rate than the lower redshift sources $(z \lesssim 2)$.

The luminosity arising in the far-infrared is a considerable fraction of the AGN bolometric luminosity ( $L_{\mathrm{bol}}$, between infrared and X-ray energies, so between $10^{12}$ and $10^{19} \mathrm{~Hz}$ ) which is typically $2 \times 10^{13} L_{\odot}$ for our sample (see Section 4 ). Hence, $L_{\mathrm{FIR}}$ is typically $\sim 0.1-0.3 L_{\mathrm{bol}}$ depending on the assumed gray body. This is slightly higher than what is found in lowredshift unobscured quasars, where $L_{\mathrm{FIR}} \lesssim 0.1 L_{\text {bol }}$ (e.g., Sanders et al. 1989; Elvis et al. 1994; Schweitzer et al. 2006, and see also Section 7). For SMGs, $L_{\mathrm{FIR}} \approx L_{\mathrm{bol}}$ since the far-infrared luminosity constitutes the bulk of the bolometric emission (e.g., Egami et al. 2004).

In addition to the data at $1.2 \mathrm{~mm}$, we have access to data at 70 and $160 \mu \mathrm{m}$ for all 21 sources (from Frayer et al. 2006), data at 350 for two sources (AMS13 and AMS16, see Section 2.2),
Table 4

Summary of Gray Body Fits

\begin{tabular}{lcccc}
\hline \hline Source & $\beta$ & $\begin{array}{c}T \\
(\mathrm{~K})\end{array}$ & $\log _{10}\left[L_{\mathrm{FIR}} / L_{\odot}\right]$ & $\log _{10}\left[M_{\mathrm{d}} / M_{\odot}\right]$ \\
\hline AMS08 $^{\mathrm{a}}$ & 1.1 & 57 & 13.0 & 8.9 \\
AMS12 $^{\mathrm{b}}$ & 1.5 & 52 & 13.4 & 9.0 \\
AMS19 $^{\mathrm{b}}$ & 1.5 & 42 & 12.9 & 9.0 \\
\hline
\end{tabular}

Notes.

${ }^{\mathrm{a}}$ In the case of AMS08, three data points are used: 70 and $160 \mu \mathrm{m}$ as well as $1.2 \mathrm{~mm}$, so that three parameters can be fitted: $\beta, T$, and $L_{\mathrm{FIR}}$.

${ }^{\mathrm{b}}$ For these two sources, the $70 \mu \mathrm{m}$ data do not provide useful constraints, and only two parameters are fitted: $T$ and $L_{\mathrm{FIR}}$, while $\beta$ is kept fixed at a value of 1.5 .

and data at $850 \mu \mathrm{m}$ for one source (AMS11; data from Frayer et al. 2004). In most of the cases, the two fiducial gray bodies provide an acceptable fit (see Figure 5). For the sources where neither the $T=47 \mathrm{~K}$ and $\beta=1.6$ or the $T=35 \mathrm{~K}$ and $\beta=1.5$ gray bodies are appropriate, we use the data to find better fits. We only fit sources that have been detected at $1.2 \mathrm{~mm}$.

For AMS08, Figure 5 suggests that the $70 \mu \mathrm{m}$ data are dominated by the cool-dust component and not by the warm torus, so we use the data at 70 and $160 \mu \mathrm{m}$, as well as $1.2 \mathrm{~mm}$, and allow $\beta, T$, and $L_{\mathrm{FIR}}$ to vary. For AMS12 and AMS19, the $70 \mu \mathrm{m}$ data do not provide tight constraints, so $\beta$ is kept fixed at a value of 1.5 , and only $T$ and $L_{\mathrm{FIR}}$ are allowed to vary. The results are summarized in Table 4 and overplotted in Figure 5. 

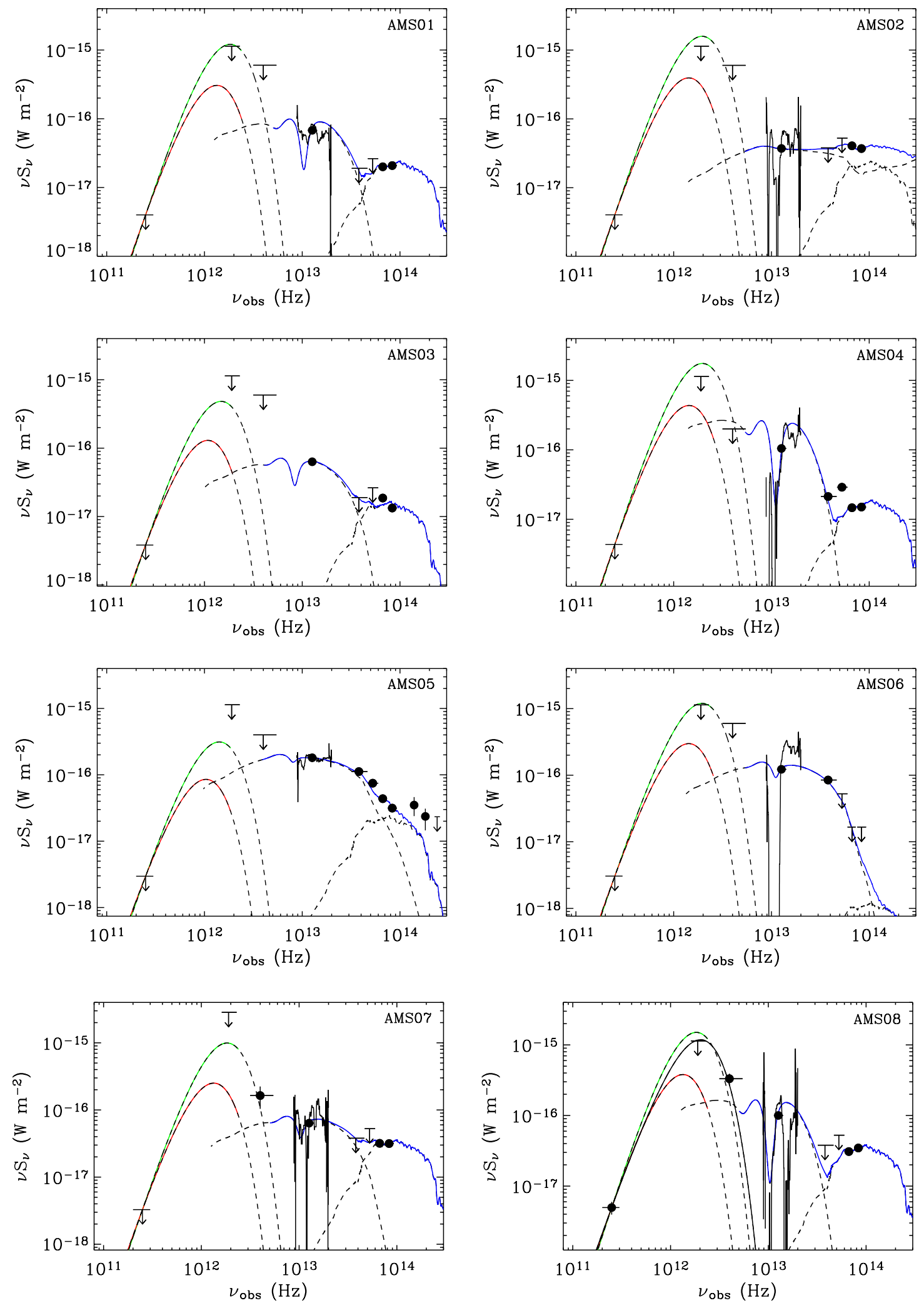

Figure 5. SEDs of the sample of high-redshift obscured quasars. Data points are marked as black points for detections or arrows for limits (left to right): $1.2 \mathrm{~mm}$, 160, 70, 24, 8, 5.8, 4.5, $3.6 \mu \mathrm{m}$ (data from Lacy et al. 2005; Fadda et al. 2006; Frayer et al. 2006, and deeper unpublished IRAC data). All limits are 3 $\sigma$. Overlaid in black are also the IRS spectra, when available. The far-infrared SED is modeled by a gray body, as described in Section 3.2. The mid-infrared component is modeled by an unobscured quasar SED with foreground extinction (see Section 4). The dashed lines are the Elvis et al. (1994) quasar SED with a screen of dust (with the value of $A_{\mathrm{V}}$ as quoted in Table 3 and using the MW dust model of Pei 1992) and a $z=0$ elliptical galaxy from Coleman et al. (1980). The quasar SED has been truncated at wavelengths longer than rest frame $80 \mu \mathrm{m}$. The blue solid line is the sum of both obscured quasar and host galaxy SEDs at wavelengths shorter than $20 \mu \mathrm{m}$, fitted using the data points between 3.6 and $24 \mu \mathrm{m}$ (see Section 4). Source AMS05 has additional data points at 1.2, 1.6, and 2.2 $\mu \mathrm{m}$ from Smith et al. (2009). Source AMS11 has an extra point at $850 \mu \mathrm{m}$, from the observations of Frayer et al. (2004), while source AMS12 has a limit on the $3 \mathrm{~mm}$ flux density from the PdBI observations. Sources AMS08, AMS12, and AMS19 have an extra gray body overplotted (black solid lines), described by the values in Table 4. Sources AMS13 and AMS16 have additional data at $350 \mu \mathrm{m}$ from the SHARC observations. In addition, AMS12 and AMS16 have a limit on the $3 \mathrm{~mm}$ flux densities from the PdBI observations. 

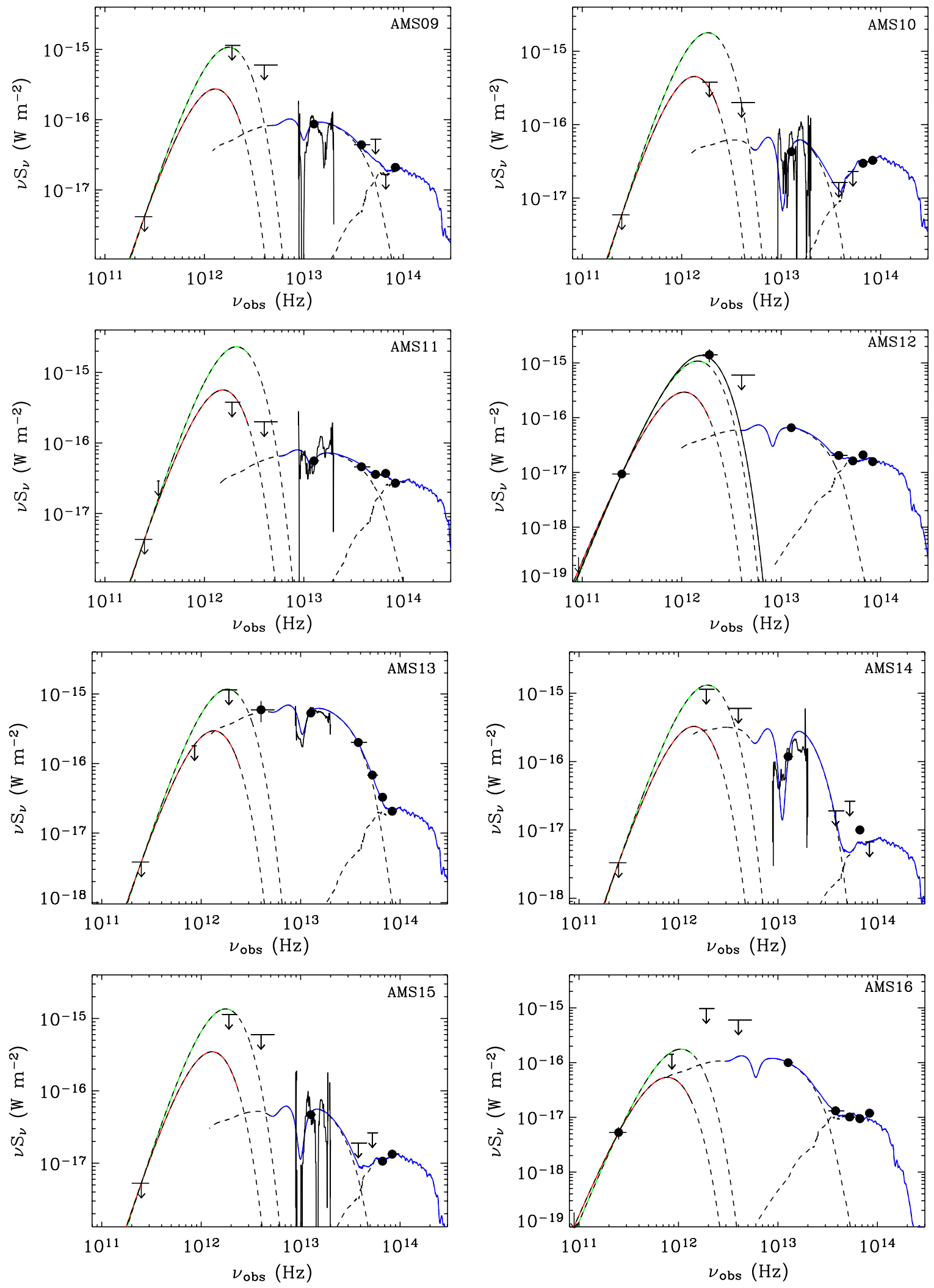

Figure 5. (Continued)

\subsection{Estimated Star Formation Rates}

Under the assumption that the far-infrared luminosity arises solely from the dust heated by young stars, and not by the AGN itself, SFRs can be estimated from $L_{\mathrm{FIR}}$. Since the dust is primarily heated by young stars, the conversion to total SFR involves assuming an initial mass function (IMF). We use the conversion from Kennicutt (1998),

$$
\left(\frac{\mathrm{SFR}}{M_{\odot} \mathrm{yr}^{-1}}\right)=1.7 \times 10^{-10}\left(\frac{L_{\mathrm{IR}}}{L_{\odot}}\right),
$$



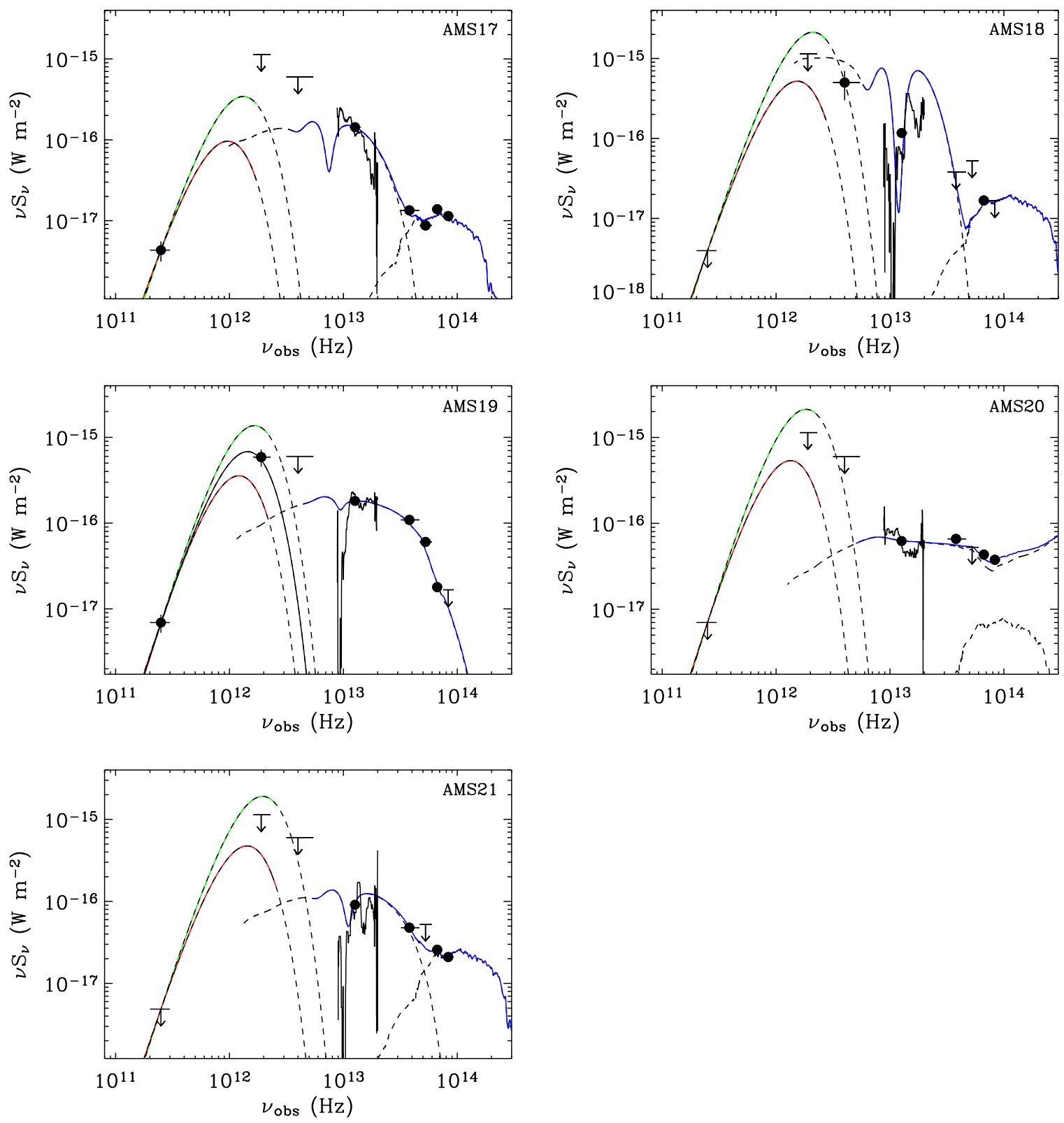

Figure 5. (Continued)

where $L_{\mathrm{IR}}$ refers to the total infrared luminosity. This conversion assumes a Salpeter (1955) IMF. Given that at mid-infrared wavelengths our sources are dominated by emission from warm dust $(T>100 \mathrm{~K})$ heated by the AGN and not by stars (see Martínez-Sansigre et al. 2008), the mid-infrared luminosities due to star formation cannot be easily derived. We therefore approximate the total infrared luminosity to the far-infrared luminosity, $L_{\mathrm{IR}} \approx L_{\mathrm{FIR}}$. $L_{\mathrm{FIR}}$ is determined by integrating analytically the fiducial gray bodies at all wavelengths, so there is no wavelength at which the integration of the gray body is cut off. However, any other components which would contribute to $L_{\mathrm{IR}}$ and are not part of the gray body (e.g., PAHs or midinfrared continuum) are neglected. Table 3 summarizes the results.

For the sources detected at $1.2 \mathrm{~mm}$, the inferred values of $L_{\mathrm{FIR}}$ and SFRs are in the range $300-3000 M_{\odot} \mathrm{yr}^{-1}$, comparable to those of SMGs (e.g., Barger et al. 1998; Hughes et al. 1998; Chapman et al. 2004; Greve et al. 2005). The SFR corresponding to $\left\langle L_{\mathrm{FIR}}\right\rangle \sim 4 \times 10^{12} L_{\odot}$ suggests that the characteristic SFR for the sample is $\sim 700 M_{\odot} \mathrm{yr}^{-1}$.

Martínez-Sansigre et al. (2008) detected polycyclic aromatic hydrocarbon (PAH) emission in three of the sources in this sample (see Table 1), and found the luminosity of the detected $7.7 \mu \mathrm{m}$ PAHs to be comparable to the typical luminosities of PAHs found in the sample of SMGs studied by Valiante et al. (2007). The far-infrared luminosities inferred here are slightly lower but still comparable to those of SMGs.

If a significant fraction of $L_{\mathrm{FIR}}$ is actually due to AGN-heated dust, these values for the SFRs will be overestimated. This could happen if dust at $\gtrsim \mathrm{kpc}$ scales is being heated by the $L_{\mathrm{bol}} \sim$ $10^{13} L_{\odot}$ quasar (see, e.g., Barvainis 1987; Sanders et al. 1989; Siebenmorgen et al. 2004; Section 3.5).

In addition to the infrared data, radio observations are also available, which could in principle yield further constraints on the SFRs. The multi-frequency and high-resolution radio data, however, suggest that the radio emission is completely AGN 
dominated, and therefore does not provide information on the SFRs (see Martínez-Sansigre et al. 2006a; Klöckner et al. 2009).

It is not possible to discriminate whether the far-infrared luminosity arises from AGN- or star-formation-heated dust: PAHs were only detected in three objects in our sample yet the upper limits obtained for the other sources correspond to very high PAH luminosities (and hence are not very stringent limits; see Martínez-Sansigre et al. 2008). The SFRs inferred for our sample are extremely high but physically possible.

\subsection{Cool-dust Masses}

The mass of cool dust responsible for the far-infrared emission can be estimated as

$$
M_{\mathrm{d}}=\frac{d_{\mathrm{L}}^{2} S_{1.2 \mathrm{~mm}}}{(1+z) \kappa\left(v_{\mathrm{rf}}\right) B_{v_{\mathrm{rf}}}\left(T, v_{\mathrm{rf}}\right)},
$$

where $\kappa$ is the mass absorption coefficient and the main source of uncertainty, so we will consider two different fiducial values to illustrate the estimated values of $M_{\mathrm{d}}$.

We follow Beelen et al. (2006) and assume $\kappa=0.04 \mathrm{~m}^{2}$ $\mathrm{kg}^{-1}$ at $1.2 \mathrm{~mm}$ (within the values quoted by Alton et al. 2004). The values for individual objects are listed in Table 3. Using $\left\langle S_{1.2 \mathrm{~mm}}\right\rangle=0.96 \pm 0.11 \mathrm{mJy}$ and again assuming $z=1.98$ as the typical redshift, we estimate the characteristic dust mass to be $\left\langle M_{\mathrm{d}}\right\rangle=2.9 \times 10^{8} M_{\odot}$ and $5.1 \times 10^{8} M_{\odot}$ (for $T=47 \mathrm{~K}$, $\beta=1.6$ and $T=35 \mathrm{~K}, \beta=1.5$, respectively). If instead we had followed Dunne et al. (2003) in assuming a value of $\kappa=$ $2.64 \mathrm{~m}^{2} \mathrm{~kg}^{-1}$ at $125 \mu \mathrm{m}$, the resulting estimates of $\left\langle M_{\mathrm{d}}\right\rangle$ would have been $=1.6 \times 10^{8} M_{\odot}$ and $2.3 \times 10^{8} M_{\odot}($ for $T=47 \mathrm{~K}$, $\beta=1.6$ and for $T=35 \mathrm{~K}, \beta=1.5$, respectively), which makes little difference to our arguments. In any case, the values of $M_{\mathrm{d}}$ quoted should be taken as indicative only, and we take $3 \times 10^{8} M_{\odot}$ as the fiducial cool-dust mass for this sample (the mean of the four values quoted above).

\subsection{Characteristic Scale of Cool Dust}

We now estimate the characteristic distance of the dust under two assumptions: that it is heated by young stars, and that it is heated by the AGN.

We note that if the dust is heated by stars, then the inferred SFRs are similar to those of SMGs. Interferometric observations of SMGs suggest a characteristic scale of $\sim 2 \mathrm{kpc}$ (Greve et al. 2005; Tacconi et al. 2006; Younger et al. 2008), so if the cool dust around our obscured quasars is heated by stars, the scale of the dust is expected to be similar to this value.

To estimate the scale of the AGN-heated dust, we equate the grain rate absorption of energy from ultraviolet photons to the radiated energy (see, e.g., Barvainis 1987, for a derivation):

$$
\frac{L_{\mathrm{uv}} e^{-\tau_{\mathrm{uv}}}}{4 \pi r^{2}}=8 \pi\left(\frac{4 \kappa_{0} a \rho}{3 v_{0}^{\beta}}\right)\left(\frac{h_{\mathrm{P}}}{c^{2}}\right)\left(\frac{k_{\mathrm{B}} T}{h}\right)^{4+\beta} \Gamma(4+\beta) \zeta(4+\beta),
$$

where $L_{\mathrm{uv}}$ is the ultraviolet luminosity of the central source, and $\tau_{\mathrm{uv}}$ is the opacity at ultraviolet wavelengths. The symbols $a$ and $\rho$ stand for the grain size and material density, and $Q(a, v)=\frac{4 \kappa_{0} a \rho}{3}\left(\frac{v}{\nu_{0}}\right)^{\beta}$ is the emissivity. Following Alton et al. (2004) we assume $a=0.1 \mu \mathrm{m}$ and $\rho=3000 \mathrm{~kg} \mathrm{~m}^{-3}$ as fiducial values, which yields:

$$
\left(\frac{r}{\mathrm{kpc}}\right)=16.3\left(\frac{T}{40 \mathrm{~K}}\right)^{-\left(\frac{4+\beta}{2}\right)}\left(\frac{L_{\mathrm{uv}} e^{-\tau_{\mathrm{uv}}}}{5 \times 10^{12} L_{\odot}}\right)^{\frac{1}{2}}
$$

The typical extinction-corrected bolometric luminosity of our sample of quasars is $2 \times 10^{13} L_{\odot}$ (see Section 4). Assuming $L_{\mathrm{uv}} \sim 0.25 L_{\mathrm{bol}}$ (e.g., Elvis et al. 1994), the typical $L_{\mathrm{uv}}$ is about $5 \times 10^{12} L_{\odot}$. If we assume the ultraviolet photons travel unhindered up to the characteristic radius of the dust, so that $\tau_{\mathrm{uv}}=0$, and assume $T=47 \mathrm{~K}$ and $\beta=1.6$, then the dust responsible for the $1.2 \mathrm{~mm}$ emission is at a distance from the AGN of $\sim 10 \mathrm{kpc}(27 \mathrm{kpc}$ for $T=35 \mathrm{~K}$ and $\beta=1.5)$.

Given that $\tau_{\mathrm{uv}}$ is likely to be $>0$ at kpc scales, since the cool dust itself can absorb the uv photons from the AGN and shield the dust on larger scales (see Section 3.6), these scales should be taken as upper limits. Hence the cool dust is expected to be distributed on scales $\lesssim 10 \mathrm{kpc}$.

\subsection{Host Obscuration?}

The characteristic cool-dust mass derived from our observations can also be used to estimate how much obscuration the host galaxy can cause. We consider the cool dust only ( $T \lesssim 50 \mathrm{~K}$ ), which emits in the far-infrared. We are interested in the amount of extinction it can cause at optical and mid-infrared wavelengths, where the emission from the cool dust is negligible. Hence, a simple screen approximation neglecting the emission from the obscuring dust is appropriate, so that a full radiativetransfer calculation can be avoided.

In order to do this, we use the dust extinction models of Pei (1992) for the Milky Way (MW) and Small Magellanic Cloud (SMC). These laws, however, correspond to $\beta=2.0$ for $\lambda \gtrsim$ $200 \mu \mathrm{m}$, so extrapolating directly from $\kappa$ at $1.2 \mathrm{~mm}$ to the visual band $(0.55 \mu \mathrm{m})$ using these extinction models would not be consistent with the values of $\beta=1.5-1.6$ we have assumed.

We therefore estimate the opacity at $200 \mu \mathrm{m}$, given a typical dust mass of $3 \times 10^{8} M_{\odot}$ assuming $\beta=1.6$. Assuming a spherically symmetric smooth distribution of dust with a characteristic radius $r$ and volume $V=\frac{4 \pi r^{3}}{3}$, the column density and hence the opacity can be estimated:

$$
\tau_{200 \mu \mathrm{m}}=r \frac{M_{\mathrm{d}}}{V} \kappa_{200 \mu \mathrm{m}}
$$

Then, using an extinction law, the corresponding extinction in the visual band, $A_{\mathrm{V}}$, can be derived (in units of magnitudes). Assuming a characteristic radius of $10 \mathrm{kpc}, \kappa=0.04 \mathrm{~m}^{2} \mathrm{~kg}^{-1}$ at $1.2 \mathrm{~mm}$, we estimate $\tau_{200 \mu \mathrm{m}}=1.2 \times 10^{-3}$, which requires $A_{\mathrm{V}}=4$ for the MW model and 1.5 for SMC. If instead we assume $\kappa=2.64 \mathrm{~m}^{2} \mathrm{~kg}^{-1}$ at $125 \mu \mathrm{m}$ and the same radius, the opacity increases to $\tau_{200 \mu \mathrm{m}}=2 \times 10^{-3}$ and requires $A_{\mathrm{V}}=7.5$ (MW) or 2.5 (SMC).

Assuming instead a radius of $2 \mathrm{kpc}$, the characteristic of the gas and dust in SMGs (Greve et al. 2005; Tacconi et al. 2006; Younger et al. 2008), the inferred value of $\tau_{200 \mu \mathrm{m}}$ is $0.03-0.05$, corresponding to values of $A_{\mathrm{V}}=35-65$ (SMC dust and depending on $\kappa$ ) or even $\geqslant 100(\mathrm{MW})$. A radius of $20 \mathrm{kpc}$ leads instead to low values of $A_{\mathrm{V}} \sim 0.3-1.5$ (using both $\mathrm{MW}$ and SMC extinction curves).

Given that the condition for a quasar to be considered obscured is $A_{\mathrm{V}} \geqslant 5$ (see Simpson et al. 1999), and comparing to the estimates of $A_{\mathrm{V}}$ from Section 4 shown in Table 3 , the main conclusion from this estimate is that there is probably enough cool dust in the host galaxies of many of these sources to obscure the quasars, regardless of the orientation of the torus with our line of sight.

There are, however, examples of high-redshift unobscured quasars with similar (sub)millimeter fluxes and hence similarly 
large dust masses (e.g., Omont et al. 2003; Beelen et al. 2006): the presence of large dust masses does not guarantee heavy obscuration. This is also the case for our sample (see Section 3.1). A likely explanation for this is that the dust in the host galaxy has a very irregular or clumpy distribution, so that only some lines of sight will be obscured. Rather than orientation-dependent obscuration, as is the case for orientation caused by the torus, the obscuration (or lack of) by dust in the host galaxy is likely to be dependent on luck, although samples of obscured quasars are expected to be biased toward "bad luck" in the form of blocked lines of sight.

\section{MID-INFRARED SED FITTING}

In this section, we make use of broadband data to characterize the SEDs of our sources. We use the IRAC and MIPS-24 $\mu \mathrm{m}$ data to obtain an estimate of $L_{\mathrm{bol}}$ and $A_{\mathrm{V}}$, from broadband SED fitting. The approximation we use is that an obscured quasar SED can be parametrized as an unobscured quasar SED, with bolometric luminosity $L_{\text {bol }}$, with a foreground screen of dust causing an amount $A_{\mathrm{V}}$ of extinction.

The values for $L_{\mathrm{bol}}$ and $A_{\mathrm{V}}$ obtained in this way are only a parametrization of the obscured SED. The $A_{\mathrm{V}}$ is only the apparent extinction, assuming the unobscured SED to be the underlying SED. This is an accurate description at optical and ultraviolet wavelengths: the intrinsic illuminating source is the accretion disk around the $\mathrm{SMBH}$, which emits thermally with a range of temperatures $\sim 10^{3}-10^{5} \mathrm{~K}$, and the dust will simply absorb this and re-emit it at longer wavelengths.

The mid-infrared emission from unobscured quasars, however, is already reprocessed light from warm dust $(T \sim 100$ $2000 \mathrm{~K})$. The dust responsible for this emission must be directly illuminated by the accretion disk, and must be relatively close to the source of ultraviolet photons: it is likely to originate in the inner region of the torus. For example, following Section 3.5 and using Equation (7), $T \sim 300 \mathrm{~K}$ corresponds to $\sim 50 \mathrm{pc}$. For an unobscured quasar, the mid-infrared emission will travel almost unhindered until it reaches the observer, while in the case of an obscured quasar, it still has to go through a considerable amount of dust. Most of this dust will be further away (and thus colder) and might not be directly illuminated by the central engine anyway. Thus, the dust obscuring the mid-infrared emission is once again unlikely to emit very significantly at the same wavelengths. The approximation of the obscured SED as an unobscured SED with a foreground screen of dust is still defendable at mid-infrared wavelengths, although it will be a poorer approximation than in the optical regime. In any case, it is a simplification of the situation.

This approximation breaks down completely in the farinfrared, so we do not attempt to model the far-infrared SEDs in this way. Instead, the far-infrared component of the SEDs was modeled as a gray body, as described in Section 3.2.

To model the SEDs in this way, we use the $24 \mu \mathrm{m}$ data from the catalog of Fadda et al. (2006), and the IRAC data from Lacy et al. (2005) as well as deeper observations around four objects from our program PID 20705 (PI: M. Lacy, the objects are AMS05, AMS12, AMS16, and AMS17). We use the Elvis et al. (1994) median quasar SED. The quasar SED is then obscured by a screen of MW-type dust, using the models of Pei (1992). It is this dust law that will determine the depth of the silicate feature (around $9.7 \mu \mathrm{m}$ ) in the models. An elliptical galaxy SED (from Coleman et al. 1980) is also used to represent the old stellar population, and this stellar light is not subject to any obscuration. The quasar bolometric luminosity, $A_{\mathrm{V}}$, and the luminosity of the galaxy are all allowed to vary, and the best fit is kept. ${ }^{13}$

For sources with many limits (namely AMS08, AMS10, AMS15, AMS18), the marginalized posterior distribution function (PDF) was flat for a range of values of $A_{\mathrm{V}}$ (i.e., the same $\chi^{2}$ was obtained for all values of $A_{\mathrm{V}}$ above a certain value). Given that low-luminosity objects are always more common than highluminosity objects, and given that the inferred value of $L_{\mathrm{bol}}$ will correlate with $A_{\mathrm{V}},{ }^{14}$ we chose the lowest value of $A_{\mathrm{V}}$ (to the closest multiple of 5) and associated $L_{\text {bol }}$ within the flat region of the posterior PDF. The results are summarized in Table 3 and all SEDs are presented in Figure 5. The best-fit SEDs are shown (blue solid line), as well as the individual components (quasar and elliptical galaxy, dashed lines). The broadband data at $3.6,4.5,5.8,8.0,24,70$, and $160 \mu \mathrm{m}$ as well as the IRS spectra are superimposed. On the far-infrared end, the $1.2 \mathrm{~mm}$ point, and the two gray bodies are also shown. For the sources AMS13 and AMS16, the $350 \mu \mathrm{m}$ data from SHARC are also shown, and for AMS11 a data point at $850 \mu \mathrm{m}$ from Frayer et al. (2004) is also included. For source AMS05, additional data at 1.2, 1.7, and $2.2 \mu \mathrm{m}$ are also shown (from Smith et al. 2009).

The IRS spectra were not used in the fit, but used to provide instead an independent quality check on the fits. In most sources the best fit to the broad-and SED is in reasonable agreement with the IRS spectrum. In a few cases, the IRS spectra show deeper silicate absorption features than those resulting from the best-fit $A_{\mathrm{V}}$ (e.g., AMS06, AMS19), in other cases the agreement is very good (e.g., AMS05, AMS13). The overall agreement between the IRS spectra and the best-fit broadband SEDs is reasonably good.

Two sources have very low values of $A_{\mathrm{V}}$ from the broadband SED fitting: AMS02 $\left(A_{\mathrm{V}}=0.5\right)$ and AMS20 $\left(A_{\mathrm{V}}=0.0\right)$. This would suggest both objects are unobscured quasars, and should not make it into the sample. However, both objects showed blank optical spectra, despite long integrations (see MartínezSansigre et al. 2005, 2006b). The IRS spectrum of AMS02 shows the silicate feature in absorption while the spectrum of AMS20 shows a hint of silicate absorption (although in Martínez-Sansigre et al. 2008, it was not considered secure due to the low signal-to-noise ratio). Both sources have $3.6 \mu \mathrm{m}$ flux densities at the edge of the selection criteria, and AMS02 also has a $24 \mu \mathrm{m}$ density at the edge of the criteria (see Table 1 and Figure 1 of Martínez-Sansigre et al. 2005). AMS02 is certainly a heavily obscured source, the flat SED might be due to a relatively bright host galaxy compared to the AGN emission. AMS20 is less clear, the flat SED could also be due to the host galaxy contribution, and in any case the blank optical spectrum shows it is certainly not an unobscured quasar. It might be a reddened quasar $\left(0<A_{\mathrm{V}} \lesssim 5\right)$, or an obscured quasar with a peculiar mid-infrared SED.

For our sample, we find no significant correlations between $L_{\mathrm{FIR}}$ and $L_{\mathrm{bol}}$ or $L_{\mathrm{FIR}}$ and $A_{\mathrm{V}}$.

\footnotetext{
13 This is essentially the same routine as used in Martínez-Sansigre et al. (2007), except the redshift is not varied and their "blue" component is not included: we use the spectroscopic redshifts, or assume $z=1.98$ when no redshift is available, while the blue component is irrelevant in the wavelength range discussed here (3.6-24 $\mu \mathrm{m})$. In addition, we are not selecting models here, only fitting parameters, so for each object we simply keep the values with the lowest $\chi^{2}$. Finally, due to the narrower wavelength range here, we allow the errors in photometry to be $<10 \%$ (c.f. Martínez-Sansigre et al. 2007). 14 In order to match the observed flux density of a given source at a given redshift, increasing the $A_{\mathrm{V}}$ will cause the inferred value of $L_{\mathrm{bol}}$ to increase.
} 


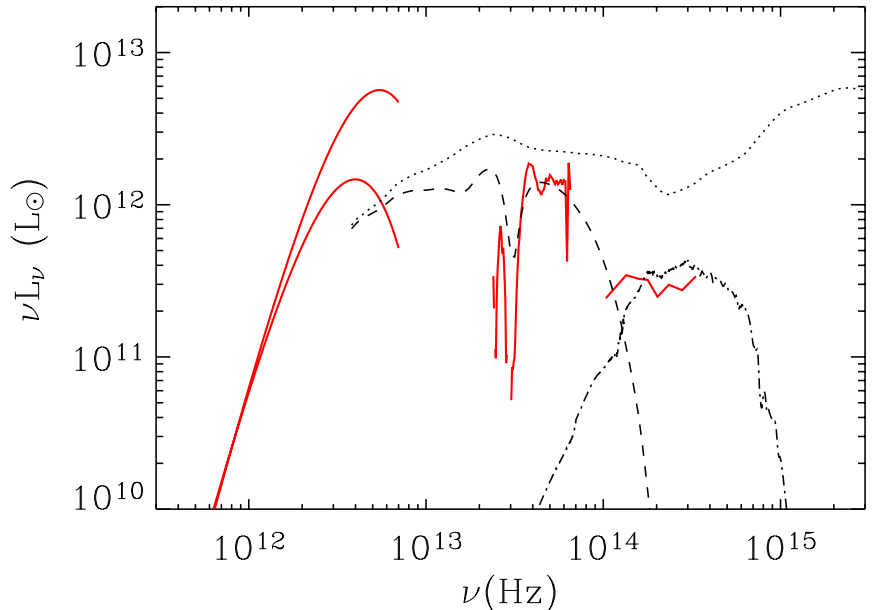

Figure 6. Mean dust SED for the sources in our sample with spectroscopic redshifts (solid red lines, see Section 5). The near-infrared component is the mean of the observed IRAC photometry $\left(v=(1-4) \times 10^{14} \mathrm{~Hz}\right.$, observed at 3.6-8.0 $\mu \mathrm{m})$, the mid-infrared component $\left(v=(2-6) \times 10^{13} \mathrm{~Hz}\right)$ is constructed using the IRS spectra, while the far-infrared component $\left(v \leqslant 1 \times 10^{13} \mathrm{~Hz}\right)$ described by either one of the two fiducial gray bodies (the top solid red line has $T=47 \mathrm{~K}, \beta=1.6$, the bottom one has $T=35 \mathrm{~K}, \beta=1.5$ ). An unobscured quasar SED (dotted line; Elvis et al. 1994) as well as the same quasar SED with a screen of MW dust (from Pei 1992) with the mean $A_{\mathrm{V}}=32$ (plotted as a dashed line), and a $z=0$ elliptical galaxy (dash-dotted line from Coleman et al. 1980) are overplotted for reference. This last SED has been normalized to be the progenitor of a present day $\sim 2 L^{\star}$ galaxy.

\section{A MEAN OBSCURED QUASAR SED}

We now derive a typical dust SED for our sample of obscured quasars and compare it to clumpy models of AGN tori.

As mentioned earlier, due to the large number of upper limits the median flux density at $1.2 \mathrm{~mm}$ is not a very useful quantity (since it yields an upper limit), yet we find the weighted mean yields a statistical detection. To be consistent, we will therefore consider mean quantities for the mid-infrared too. We note that within our sample there is no correlation between $L_{\text {bol }}$ and $L_{\mathrm{FIR}}$, so we treat the (rest-frame) near-infrared, mid-infrared, and far-infrared components independently. The mean SED of our spectroscopically confirmed objects is shown as a solid red line in Figure 6.

We do warn about several caveats: the far-infrared, midinfrared, and near-infrared components have been determined in different ways. The far-infrared component is an analytic gray body to the mean flux density at $1.2 \mathrm{~mm}$. It is still ill constrained, since only some objects have been detected at 70 or $160 \mu \mathrm{m}$. The mid-infrared component results from stacking IRS spectra, and is probably the best determined part, although it uses only sources with spectroscopic redshifts and IRS spectra.

For the entire SED, only sources with spectroscopic redshifts are used. For sources with no redshift from optical spectroscopy, redshifts can only be determined if the silicate feature is deep enough to be noticeable in noisy spectra. The mean mid-infrared SED is therefore partially biased toward sources with deep absorption features at $9.7 \mu \mathrm{m}$.

The near-infrared component uses the data from all sources, but because these sources have a variety of redshifts, of values of $A_{\mathrm{V}}$, and of host galaxy luminosities, it is really a mixture of heavily extinct AGN light and stellar light.

In addition, the sources in this sample probably include examples of both torus- and host-obscured quasars, meaning that the mean SED will represent a mixture of such objects.
Despite all these caveats, we consider the mean SED to be a useful fiducial representation of obscured quasars.

\subsection{Far-infrared Component}

We parametrize the far-infrared component as being described by the two fiducial gray bodies. The far-infrared luminosity is determined by the weighted mean flux density, $\left\langle S_{1.2 \mathrm{~mm}}\right\rangle=0.96 \pm 0.11 \mathrm{mJy}$ at $z=1.98$. Assuming the gray body with $T=47 \mathrm{~K}$ and $\beta=1.6$ results in $\left\langle L_{\mathrm{FIR}}\right\rangle=6.3 \times$ $10^{12} L_{\odot}$, while assuming $T=35 \mathrm{~K}$ and $\beta=1.5$, we obtain $\left\langle L_{\mathrm{FIR}}\right\rangle=1.6 \times 10^{12} L_{\odot}$.

\subsection{Mid-infrared Component}

The mid-infrared component was obtained by shifting the IRS spectra to rest-frame wavelengths, interpolating to a common wavelength grid and taking the mean luminosity density of the 14 sources with spectroscopic redshifts and mid-infrared spectra. Different wavelength bins have a different total number of sources contributing, and it was decided to cut below $4.5 \mu \mathrm{m}$ and above $12.5 \mu \mathrm{m}$, where three or less sources were contributing. Figure 6 shows the mean IRS spectrum smoothed by a box-car average of $0.7 \mu \mathrm{m}$.

The depth of the silicate absorption feature at $9.7 \mu \mathrm{m}, \tau_{9.7}$, is usually measured by interpolating the continuum at $9.7 \mu \mathrm{m}$ using the continuum blueward and redward. The difference in flux densities between the interpolated continuum $\left(f_{\text {IC }}\right)$ and the observed feature $\left(f_{\mathrm{OF}}\right)$ then yields $\tau_{9.7}$ :

$$
\tau_{9.7} \equiv \ln \left(\frac{f_{\mathrm{IC}}}{f_{\mathrm{OF}}}\right) .
$$

This is difficult to measure from the mean IRS spectrum, since there are no data points redward of the absorption feature, so no real interpolation can be made. However, comparing the flux densities of the $9.7 \mu \mathrm{m}$ feature and the $8 \mu \mathrm{m}$ continuum in Figure 6, the depth of the feature is likely to be in the range $2.5 \lesssim \tau_{9.7} \lesssim 3.0$

Figure 6 also shows that this is deeper than what is expected from the unobscured SED with the screen of dust (using the mean $A_{\mathrm{V}}$ : dashed line), which otherwise fits the region of 4.5$8.5 \mu \mathrm{m}$ quite well. We note that the mean $A_{\mathrm{V}}$ from all sources with spectroscopic redshifts $\left(A_{\mathrm{V}}=32\right.$ is very similar to the mean of the 14 sources used for the mid-infrared component, $A_{\mathrm{V}}=$ 34). This small difference leads to a practically indistinguishable SED in the mid-infrared and cannot explain the discrepancy in the depth of the silicate feature.

At this stage, the discrepancy with the silicate feature is only due to the dust extinction model used: an extinction law with deeper silicate absorption (such as the SMG dust model of Pei et al. 1992) can plausibly reproduce the observed feature.

However, a typical silicate feature as deep as $\gtrsim 2.5$ suggests some degree of geometrical obscuration caused by cold dust (see Levenson et al. 2007, for a discussion). This lends further support for some degree of host obscuration.

Finally, the mean spectrum from the IRS shows an absorption feature at $\sim 7 \mu \mathrm{m}$ probably due to hydrogenated amorphous carbons (see, e.g., Spoon et al. 2002).

\subsection{Near-infrared Component}

For the near-infrared component, for each object the flux densities of the IRAC bands were converted to luminosities at the rest-frame wavelength. They were then binned in a grid 

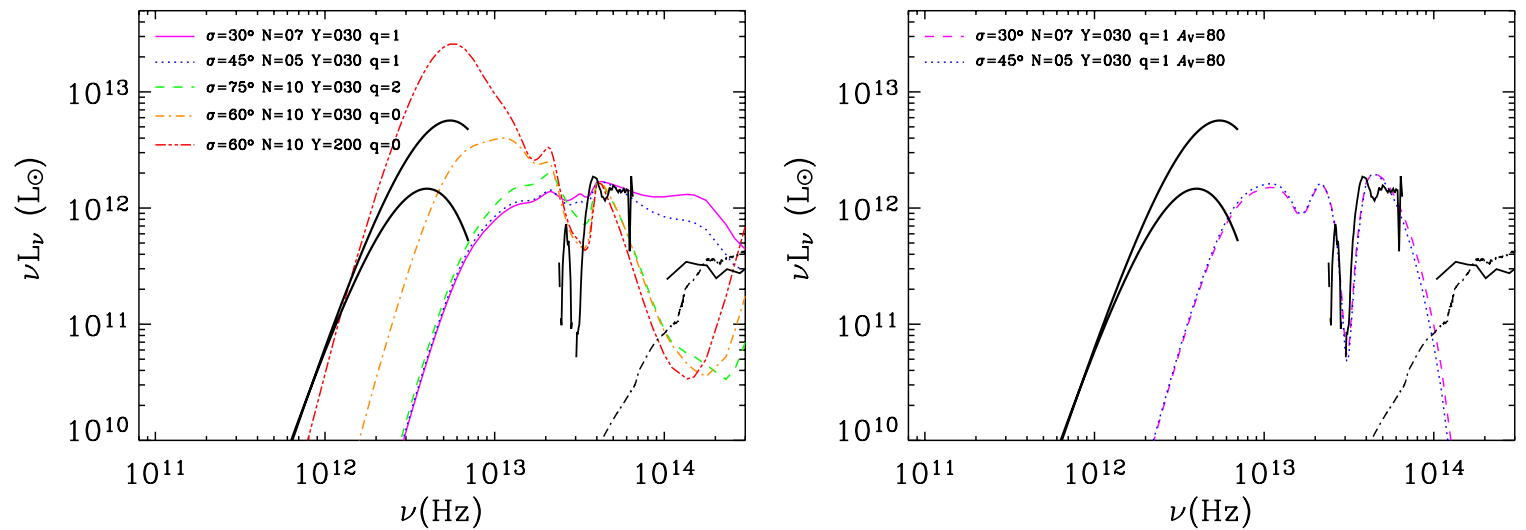

Figure 7. Comparison of the mean SED (solid black lines) with different clumpy models of AGN tori. The progenitor of a $2 L^{\star}$ galaxy has been overplotted again (dot-dashed line). The clumpy models, from Nenkova et al. (2008a, 2008b), all have single-cloud optical depths $\tau_{\mathrm{V}}=60$. Left panel: different lines show models with varying parameters for the width of the Gaussian angular distribution of clumps around the equator, $\sigma$, the number of clouds along a ray in the equatorial plane $N$, the ratio of inner to outer torus radius $Y$, and the power of the radial density distribution, $q$. The models cannot simultaneously reproduce the relatively flat continuum between $1 \times 10^{13}$ and $7 \times 10^{13} \mathrm{~Hz}$, the sudden drop at higher frequencies, the depth of the silicate feature, and the millimeter detection. Right panel: additional extinction from dust along the line of sight has been added to the two flatter clumpy models from the left panel. The emission from this cool dust is represented by the two fiducial gray bodies used throughout this work. The typical quasar SED of our sample can be reasonably reproduced using the clumpy torus models, with foreground extinction $A_{\mathrm{V}}=80$ and re-emission from a mass of $3 \times 10^{8} M_{\odot}$ of cool dust. The emission from this cool-dust mass and the foreground extinction is consistent provided the dust is within a $2 \mathrm{kpc}$ radius (using Equation (8) from Section 3.6).

ranging between 0.5 and $4.5 \mu \mathrm{m}$. Looking at the SEDs in Figure 5 it seems that the upper limits are generally close to the detections in adjacent bands, so we treat limits like detections. In the bins with contributions from several sources, the mean luminosity density was computed. However, some of the bins have a contribution from only one object and some from none (these last bins are not shown in the plots). The solid red line in Figure 6 shows the mean photometry at rest-frame near-infrared, smoothed by a box-car average of $0.6 \mu \mathrm{m}$. The line is irregular, because of the vastly different individual SEDs seen in Figure 5: in some objects, all IRAC bands are likely to be dominated by the AGN (e.g., AMS19), in others by the host galaxy (e.g., AMS16). If instead of using limits as data, we do not use them at all, the resulting SED is very similar.

This part of the SED should clearly be treated with caution. However, an estimate of the luminosity of the typical host galaxy can be attempted. In Figure 6, a $z=0$ elliptical galaxy from Coleman et al. (1980), normalized to be 12.6 times brighter than an $L^{\star}$ galaxy in the local $K$-band luminosity function (Cole et al. 2001), is overplotted. Assuming 2 mag of passive evolution between $z \sim 2$ and $z=0$, it is equivalent to a present day $2 L^{\star}$ galaxy (this is partly by selection, as is described in detail by Martínez-Sansigre et al. 2005, 2006b). We can thus see that the luminosity of the near-infrared component of the typical obscured quasar SED corresponds approximately to the progenitor of a $2 L^{\star}$ galaxy, although it might also have an important contribution from AGN light (in which case the galaxy luminosity will be overestimated).

\subsection{Comparison of Clumpy Models of the Torus}

It is of particular interest to compare the mean SED with clumpy models of the AGN torus. This is shown in Figure 7 (left panel), where the mean SED is overplotted on the models of Nenkova et al. (2008a, 2008b). The models cannot reproduce simultaneously the relatively flat SED at wavelengths shorter than $8 \mu \mathrm{m}$, the sharp drop in the near-infrared and the deep silicate absorption. The left panel in Figure 7 also shows that the models with deeper silicate features still struggle to match the observed silicate depth $\left(\tau_{9.7} \gtrsim 2.5\right)$ yet will overpredict the far-infrared luminosity.
The right panel in Figure 7 shows the two flattest clumpy SEDs from the right panel, now with an additional extinction of $A_{\mathrm{V}}=80$ added due to foreground dust.

We see that the clumpy models, with the additional extinction and re-emission from the cool dust can reproduce reasonably well the observed SED. Since the dust is cold, it re-emits in the far-infrared, meaning it does not "fill-up" the silicate absorption feature, unlike dust at $\sim 300 \mathrm{~K}$ would (again, see Levenson et al. 2007). To reproduce the deep silicate feature, a value of $A_{\mathrm{V}} \sim$ 80 is required (see Figure 7).

A typical dust mass of $3 \times 10^{8} M_{\odot}$ can cause this extinction of $A_{\mathrm{V}}=80$ provided that the dust is on scales $<2.3-3.0 \mathrm{kpc}$ (from Equation (8) in Section 3.6 and assuming MW dust and the two values of $\kappa_{200 \mu \mathrm{m}}$ ). This is in excellent agreement with the inferred scales of the cool-dust emission in SMGs (typically $\sim 2$ kpc Greve et al. 2005; Tacconi et al. 2006; Younger et al. 2008).

Polletta et al. (2008) studied in detail the mid-infrared spectra and mid- to near-infrared SEDs of 21 high-redshift heavily obscured quasars, selected to be bright $(\geqslant 1 \mathrm{mJy})$ at $24 \mu \mathrm{m}$, optically faint (typically $R>24$ ), and with mid-infrared spectra characteristic of heavily obscured AGN (see their Section 2 for more details). They found that nine out of 21 sources did not fit clumpy models well and required an additional foreground screen of dust that did not re-emit at mid- or near-infrared wavelengths.

Our findings for the mean SED are consistent with their results, and in addition, we are able to estimate the $A_{\mathrm{V}}$ in a consistent way from $M_{\mathrm{d}}$, which was derived from our observations at $1.2 \mathrm{~mm}$. The resulting dust size required for $M_{\mathrm{d}}$ to cause the $A_{\mathrm{V}}(\sim 2.3-3.0 \mathrm{kpc})$ is very close to the sizes estimated for the far-infrared emission of SMGs. Again, our results provide evidence for obscuration due to dust on kpc scales, dust that has lower temperatures and is distributed on larger scales than what is expected for the dust of the torus.

\subsection{Comparison to Local ULIRGs}

Figure 8 shows the mid-infrared component of the mean SED with the spectra of four local ultra-luminous infrared galaxies overplotted (ULIRGs, with $L_{\mathrm{IR}}>10^{12} L_{\odot}$ ). The spectra for 


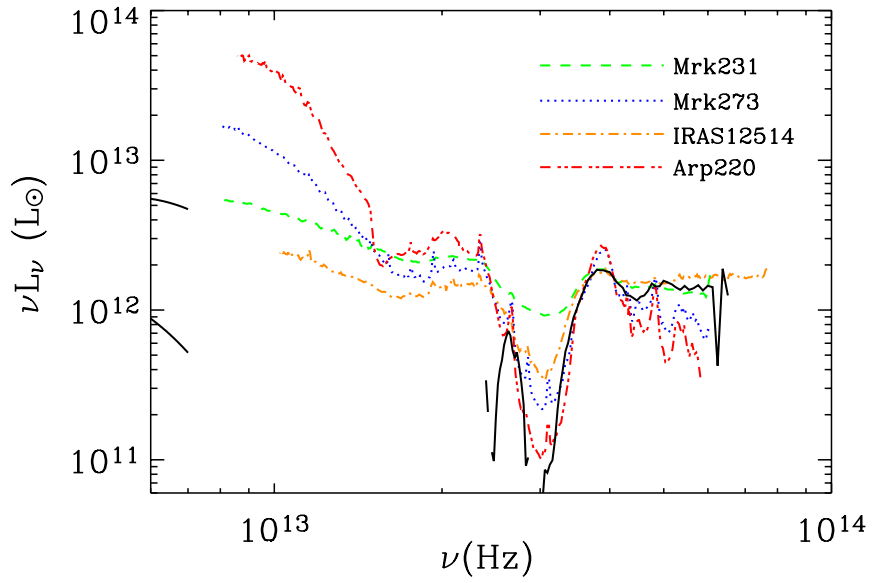

Figure 8. Comparison of the mid-infrared component of the mean SED (black solid line) with four local ULIRGs: IRAS 12514 + 1027 (hereafter IRAS 12514), Mrk 231, Mrk 273, and Arp 220.

these sources were obtained from Armus et al. (2007) and Spoon et al. (2007). These ULIRGs represent one pure-AGN, IRAS 12514 + 1027 (hereafter IRAS 12514), two AGN-starburst composites, Mrk 231 and Mrk 273, and one starburst-dominated source, Arp 220. All spectra have been normalized to match our mean SED between 7.0 and $7.8 \mu \mathrm{m}$ (around $4 \times 10^{13} \mathrm{~Hz}$ ).

Compared to these sources, our mean SED is relatively flat, similarly to Mrk 231 or IRAS 12514, yet the silicate absorption feature is significantly deeper, more similar to that of Mrk 273 or even Arp 220. Once again, we see the peculiar spectral shape of this mean SED, where the silicate feature is suprisingly deep given the relative flatness of the continuum. The most similar nearby ULIRG is IRAS 12514 , which is known to be an obscured quasar (Wilman et al. 2003), although this source has a shallower feature at $9.7 \mu \mathrm{m}$.

\section{RESULTS FROM OBSERVATIONS OF CO}

\subsection{Line Properties}

Two sources, AMS12 and AMS16, were observed with the IRAM PdBI to search for the CO (3-2) or (4-3) rotational transitions, tracers of molecular gas.

The CO (3-2) line in AMS12 is clearly detected, and is well described by a Gaussian with a peak flux of 2.14 mJy and a fullwidth half-maximum (FWHM) of $275 \mathrm{~km} \mathrm{~s}^{-1}$ (see Figure 9). The integrated line flux of this Gaussian is $S_{\mathrm{CO}} \Delta v=630 \pm$ $50 \mathrm{mJy} \mathrm{km} \mathrm{s}{ }^{-1}$, where $S_{\mathrm{CO}} \Delta v$ is the velocity-integrated CO line flux. This corresponds to a (frequency-integrated) luminosity of $L_{\mathrm{CO}(3-2)}=3.2 \times 10^{7} L_{\odot}$, or a brightness-temperature luminosity of $L_{\mathrm{CO}(3-2)}^{\prime}=2.4 \times 10^{10} \mathrm{~K} \mathrm{~km} \mathrm{~s}^{-1} \mathrm{pc}^{2}$.

The central frequency of the line is at $-16 \mathrm{~km} \mathrm{~s}^{-1}$, corresponding to a frequency shift of $4.9 \mathrm{MHz}$ (so a central frequency of $91.801 \mathrm{GHz}$ ), so the redshift of the $\mathrm{CO}$ line is $z_{\mathrm{CO}}=2.7668$. At our signal to noise, the line shape is well described by a Gaussian, there is no double profile visible, which could suggest a disk. There is also no hint of two separate lines which could suggest that the molecular gas is undergoing a major merger.

In the case of AMS16, no line was detected (see Figure 10). The full data cube was searched by looking for line emission at various spectral resolutions, using a routine provided by Roberto Neri, but no significant emission was detected at the position of the source.

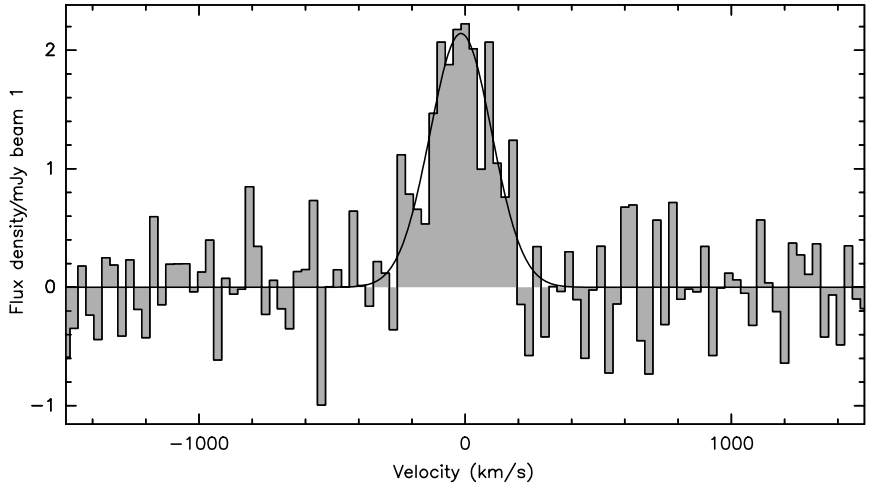

Figure 9. Spectrum of AMS12 obtained with the Plateau de Bure Interferometer The line is well fitted by a single Gaussian centered on $-16 \mathrm{~km} \mathrm{~s}^{-1}\left(z_{\mathrm{CO}}=\right.$ 2.7668 ) with an FWHM of $275 \mathrm{~km} \mathrm{~s}^{-1}$. From this Gaussian fit, the $\mathrm{CO}(3-2)$ line has an integrated flux of $630 \mathrm{mJy} \mathrm{km} \mathrm{s}^{-1}$ corresponding to a frequencyintegrated line luminosity of $L_{\mathrm{CO}(3-2)}=3.2 \times 10^{7} L_{\odot}$, or a brightnesstemperature luminosity of $L_{\mathrm{CO}(3-2)}^{\prime}=2.4 \times 10^{10} \mathrm{~K} \mathrm{~km} \mathrm{~s}^{-1} \mathrm{pc}^{2}$.

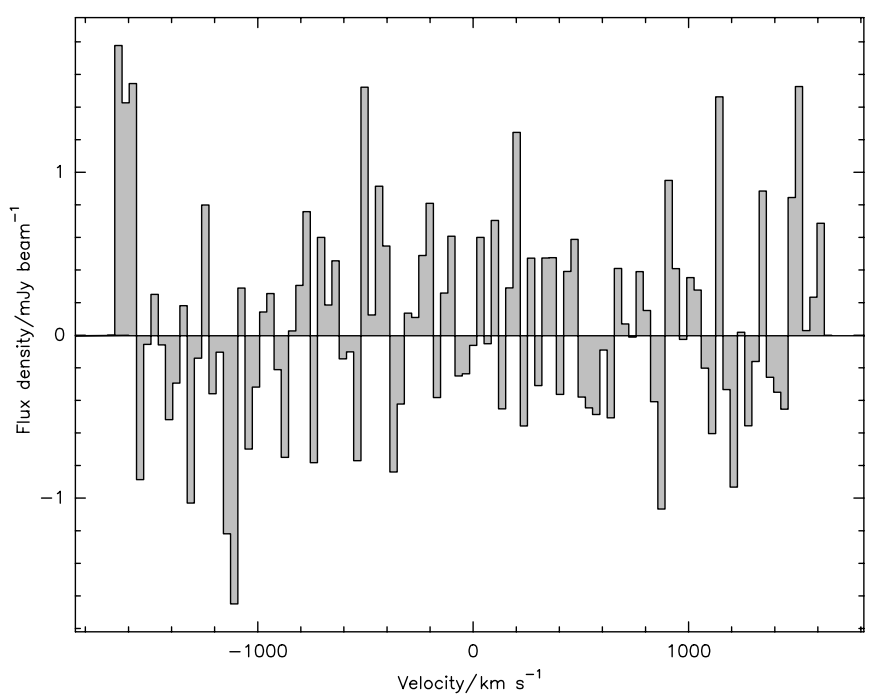

Figure 10. Spectrum of AMS16 obtained with the Plateau de Bure Interferometer. The frequency was centered on $89.2002 \mathrm{GHz}$, the expected location of the $\mathrm{CO}(4-3)$ rotational transition. The $\mathrm{rms}$ noise per $10 \mathrm{MHz}$ bin $\left(33 \mathrm{~km} \mathrm{~s}^{-1}\right)$ is $0.65 \mathrm{mJy}$ beam $^{-1}$, assuming a box-car CO line shape with full width to a zero intensity of $400 \mathrm{~km} \mathrm{~s}^{-1}$ and the line to be within the frequency covered, the $3 \sigma$ limit is $\Delta v S_{\mathrm{CO}}<230 \mathrm{mJy}$ beam $^{-1} \mathrm{~km} \mathrm{~s}^{-1}$, or $L_{\mathrm{CO}(4-3)}^{\prime}<1 \times 10^{10} \mathrm{~K} \mathrm{~km} \mathrm{~s}^{-1}$ $\mathrm{pc}^{2}$ at the redshift of the source.

A signal at the $4.5 \sigma$ level can be seen at $171944+584700$. If real this would correspond to a line offset from the narrow-line redshift by $\sim 600 \mathrm{~km} \mathrm{~s}^{-1}$ and with a full width at zero intensity of $\sim 850 \mathrm{~km} \mathrm{~s}^{-1}$. However, the position does not correspond to any source detectable in the $R$-band, $3.6 \mu \mathrm{m}$ or $1.4 \mathrm{GHz}$ image. This position corresponds to a 17 arcsec offset, or $116 \mathrm{kpc}$ at the redshift of the source. We do not consider this emission any further.

The lack of detection of the $\mathrm{CO}$ (4-3) line can either be due to the line being shifted outside of the observed bandwidth ( $> \pm 1680 \mathrm{~km} \mathrm{~s}^{-1}$ shift), or the line being within the bandwidth but too faint. The redshift was determined from three

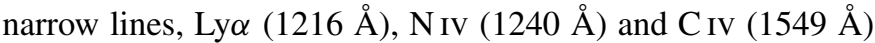
with individual redshifts of $z=4.171,4.166$, and 4.170, respectively (see Martínez-Sansigre et al. 2006a). Thus the maximum redshift offset of any individual line, with respect to 
the average $\langle z\rangle=4.169$, is that of the $\mathrm{N}$ Iv line with $\Delta z=0.003$, which only corresponds to a $175 \mathrm{~km} \mathrm{~s}^{-1}$ offset.

Given the bandwidth used, we consider the possibility that the $\mathrm{CO}$ (4-3) line falls outside of the observed range unlikely, so detection of CO (4-3) in AMS16 can be ruled out down to a $3 \sigma$ limit of $S_{\mathrm{CO}} \Delta v<0.23 \mathrm{Jy} \mathrm{km} \mathrm{s}^{-1}$, assuming a box-car line shape with full width to a zero intensity of $400 \mathrm{~km} \mathrm{~s}^{-1}$. From these limits, we now estimate the limits on the CO (4-3) luminosities and derived molecular gas properties. Figure 10 shows the spectrum extracted at the position of AMS16.

At $z=4.169, S_{\mathrm{CO}} \Delta v<230 \mathrm{mJy} \mathrm{km} \mathrm{s}{ }^{-1}$ corresponds to a frequency-integrated line luminosity of $L_{\mathrm{CO}(4-3)}<3 \times 10^{7} L_{\odot}$, or a brightness-temperature luminosity of $L_{\mathrm{CO}(4-3)}^{\prime}<1 \times 10^{10} \mathrm{~K}$ $\mathrm{km} \mathrm{s}^{-1} \mathrm{pc}^{2}$.

\subsection{Inferred Physical Properties}

If we assume that the molecular gas responsible for the $\mathrm{CO}$ emission is dense enough and warm enough for the (3-2) or (43) transitions to be thermalized, then the brightness-temperature luminosities of these high- $J$ transitions are the same as that of the (1-0) transition: $L_{\mathrm{CO}(1-0)}^{\prime}=L_{\mathrm{CO}(3-2)}^{\prime}$ and $L_{\mathrm{CO}(1-0)}^{\prime}=L_{\mathrm{CO}(4-3)}^{\prime}$.

Following Downes \& Solomon (1998), we can then estimate an upper limit on the mass of molecular hydrogen, by using

$$
\left(\frac{M_{\mathrm{H}_{2}}}{M_{\odot}}\right)=\alpha_{\mathrm{CO}}\left(\frac{L_{\mathrm{CO}(1-0)}^{\prime}}{L_{\odot}}\right),
$$

where $\alpha_{\mathrm{CO}}=0.8\left(\mathrm{~K} \mathrm{~km} \mathrm{~s}^{-1} M_{\odot}\right)^{-1}$ is the conversion factor for local ULIRGs (Downes \& Solomon 1998). Thus, under the assumption that $L_{\mathrm{CO}(1-0)}^{\prime}=L_{\mathrm{CO}(3-2)}^{\prime}$, we infer a total gas mass of $M_{\mathrm{H}_{2}}=1.9 \times 10^{10} M_{\odot}$ for AMS12. Assuming that $L_{\mathrm{CO}(1-0)}^{\prime}=L_{\mathrm{CO}(4-3)}^{\prime}$, we can derive a limit for the total $\mathrm{H}_{2}$ mass in AMS16 of $M_{\mathrm{H}_{2}}<8 \times 10^{9} M_{\odot}$.

The far-infrared luminosity of AMS12 of $2.5 \times 10^{13} L_{\odot}$ (see Table 4) suggests an SFR of $4300 M_{\odot} \mathrm{yr}^{-1}$. The ratio of far to CO luminosity is $\log _{10}\left[L_{\mathrm{FIR}} / L_{\mathrm{CO}}^{\prime}\right]=3.0$, the gas-to-dust ratio, $M_{\mathrm{g}} / M_{\mathrm{d}}$, is 19 , and the gas depletion timescale is estimated to be $\tau_{\mathrm{g}} \sim 4 \mathrm{Myr}$.

For AMS16, we can only infer limits for these values: if we assume the values from the $T=35 \mathrm{~K}, \beta=1.5$ gray body, we infer $\log _{10}\left[L_{\mathrm{FIR}} / L_{\mathrm{CO}}^{\prime}\right]>2.4, M_{\mathrm{g}} / M_{\mathrm{d}}<8$, and $\tau_{\mathrm{g}}<16 \mathrm{Myr}$. Assuming instead the $T=47 \mathrm{~K}, \beta=1.6$ gray body yields the following values: $\log _{10}\left[L_{\mathrm{FIR}} / L_{\mathrm{CO}}^{\prime}\right]>2.9, M_{\mathrm{g}} / M_{\mathrm{d}}<20$, and $\tau_{\mathrm{g}}<5 \mathrm{Myr}$.

For both AMS12 and AMS16, the estimated values of $\log _{10}\left[L_{\mathrm{FIR}} / L_{\mathrm{CO}}^{\prime}\right]$ are at the low end but consistent with the observed values for other high-redshift sources with molecular gas measurements (e.g., Solomon \& Vanden Bout 2005; Greve et al. 2005). The gas-to-dust ratios are much lower than the values $\sim 100-150$ found for local spiral galaxies. The values of $\tau_{\mathrm{g}}$ are ultimately derived from the quantity $L_{\mathrm{FIR}} / L_{\mathrm{CO}}^{\prime}$ and are thus also at the low end of the observed values. A possible explanation for the high values of $L_{\mathrm{FIR}} / L_{\mathrm{CO}}^{\prime}$ is that a fraction of the far-infrared luminosity is due to AGN-heated dust, rather than by dust heated by young stars.

We warn that if the assumption that the (4-3) transition is thermalized is inappropriate, $L_{\mathrm{CO}(1-0)}^{\prime}>L_{\mathrm{CO}(3-2)}^{\prime}$ or $L_{\mathrm{CO}(1-0)}^{\prime}>$ $L_{\mathrm{CO}(4-3)}^{\prime}$, we will have underestimated $M_{\mathrm{g}}$, and hence $\tau_{\mathrm{g}}$ and the gas-to-dust ratio. For a discussion about whether this assumption is appropriate, see Papadopoulos et al. (2001) and Riechers et al. (2006), although for the high-redshift AGN studied in detail it seems to be a reasonable assumption (see, e.g., Beelen et al. 2004; Klamer et al. 2005; Aravena et al. 2008).
From the FWHM of the $\mathrm{CO}(3-2)$ line in AMS12, $\Delta v=$ $275 \mathrm{~km} \mathrm{~s}^{-1}$, and assuming a characteristic radius, we can estimate the dynamical mass given (see Neri et al. 2003):

$$
\left(\frac{M_{\mathrm{dyn}} \sin ^{2} i}{M_{\odot}}\right)=4 \times 10^{4}\left(\frac{\Delta v}{\mathrm{~km} \mathrm{~s}^{-1}}\right)^{2}\left(\frac{r}{\mathrm{kpc}}\right) .
$$

The CO is unresolved in the $\sim 5$ arcsec resolution observations with the PdBI observations, yielding a size limit of $r<40 \mathrm{kpc}$. A better estimate for $r$ is $2 \mathrm{kpc}$ (Tacconi et al. 2006). The inferred mass is then $M_{\mathrm{dyn}} \sin ^{2} i=6 \times 10^{9} M_{\odot}$, and is obviously dependent on the unknown inclination angle $i$. The ratio of gas to dynamical mass is then $M_{\mathrm{H}_{2}} / M_{\mathrm{dyn}}=3 \times \sin ^{2} i$. Thus the estimated gas mass is larger than the estimated dynamical mass, unless the inclination angle $i$ is $\leqslant 35$. It seems likely that the gas mass represents a high fraction of the total dynamical mass, as found for other high-redshift galaxies observed in $\mathrm{CO}$ (Greve et al. 2005; Tacconi et al. 2006).

\section{COMPARISON TO OTHER SAMPLES OF $z \sim 2$ QUASARS}

Other samples of $z \sim 2$ quasars have been observed at millimeter or submillimeter wavelengths, allowing us to compare the far-infrared luminosities inferred for the different samples. Far-infrared emission being isotropic, the properties of obscured and unobscured quasars should be similar if obscuration is only an orientation effect. To test this, we consider here three other studies at approximately the same redshift:

1. The sample of Omont et al. (2003, hereafter O03), consisting of optically selected unobscured quasars, observed at $1.2 \mathrm{~mm}$. These sources are intrinsically very bright with $M_{\mathrm{B}} \lesssim-27$, corresponding to $L_{\mathrm{bol}}$ in the range $5 \times 10^{13}$ to $5 \times 10^{14} L_{\odot}$, and at $1.94 \leqslant z \leqslant 2.85$. They have a detection rate of nine out of 26 sources (35\%).

2. The sample presented by Page et al. (2004, P04), which consists of optically unobscured quasars known also to be X-ray unabsorbed. These sources have values of $L_{\mathrm{bol}} \sim$ $10^{13} L_{\odot}$, similar to our sample, with $1.20 \leqslant z \leqslant 2.35$. They were observed at $850 \mu \mathrm{m}$, with a detection rate of one out of $20(5 \%)$.

3. The optically unobscured but X-ray-absorbed quasars from the sample of Stevens et al. (2005, S05), with similar values of $L_{\text {bol }}$ to our sample, $1.01 \leqslant z \leqslant 2.80$. They were observed at $850 \mu \mathrm{m}$ with six out of 19 sources detected at the $\geqslant 3 \sigma$ level (32\%).

In all samples, the bolometric luminosities have been estimated consistently using the Elvis et al. (1994) SED, and correcting for absorption at X-ray energies (S05) or dust extinction at optical wavelengths (this sample). The far-infrared luminosities have all been computed assuming a gray body with $T=$ $47 \mathrm{~K}, \beta=1.6$. Changing these parameters will affect very similarly the far-infrared luminosities of all samples.

Figure 11 shows $L_{\mathrm{FIR}}$ versus $L_{\mathrm{bol}}$ for the four samples of $z \sim 2$ quasars. The colored symbols represent the individual sources, the black filled symbols the mean values for each sample, and the black empty symbols the mean of the non-detections. The dashed line shows a constant far-infrared fraction of $\frac{L_{\mathrm{FIR}}}{L_{\mathrm{bol}}}=0.05$, characteristic of the low-redshift quasars studied by Elvis et al. (1994).

From the available data, the $\mathrm{O} 03$ and $\mathrm{P} 04$ samples as a whole have a ratio of $\frac{L_{\mathrm{FIR}}}{L_{\mathrm{bol}}}$ similar to the low-redshift quasars. However, 


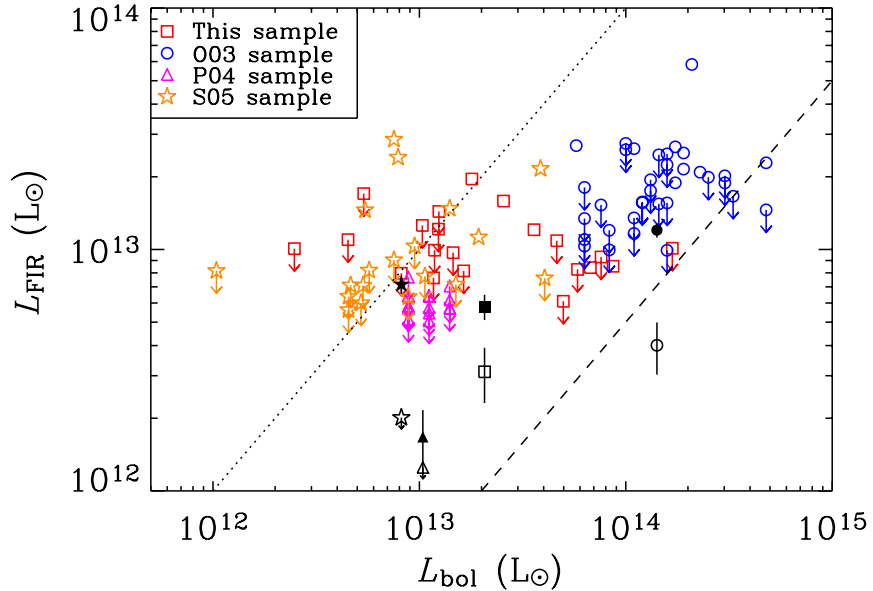

Figure 11. $L_{\mathrm{FIR}}$ vs. $L_{\mathrm{bol}}$ for the four samples of $z \sim 2$ quasars considered. $\mathrm{O} 03$ is the sample presented by Omont et al. (2003), P04 by Page et al. (2004), and S05 by Stevens et al. (2005). Black filled symbols represent the weighted mean of all sources in each sample, black empty symbols represent the weighted mean of non-detections in each sample. The dashed line represents $L_{\mathrm{FIR}}=0.05 L_{\mathrm{bol}}$, the value found in low-redshift quasars considered by Elvis et al. (1994). For reference, the line $L_{\mathrm{FIR}}=L_{\mathrm{bol}}$ has been plotted as a dotted line. For all samples, a gray body with $T=47 \mathrm{~K}$ and $\beta=1.6$ has been assumed to calculate $L_{\mathrm{FIR}}$.

our sample and the S05 samples both seem to have larger farinfrared fractions than the $\mathrm{O} 03$ and $\mathrm{P} 04$ samples. ${ }^{15}$

If all obscuration were orientation dependent then the host galaxies of obscured and unobscured quasars would be expected to have, on average, the same properties, such as SFRs (e.g., Clavel et al. 2000) and cool-dust content (e.g., Haas et al. 2004). If, however, some obscured quasars are at a different evolutionary stage, then their host galaxy can be reasonably expected to have a larger gas and dust content, which might be causing the obscuration, or at least contributing to it. Obscured quasars might therefore be in a different evolutionary phase to unobscured quasars (e.g., Sanders et al. 1988; Fabian 1999).

The extinction due to dust is negligible in the far-infrared (FIR), so that at wavelengths $\gtrsim 50 \mu \mathrm{m}$ the emission becomes essentially isotropic. Hence, if orientation is the only difference between obscured and unobscured quasars, they should have virtually identical cool-dust properties, irrespective of whether this emission is due to dust heated by the quasar or by young stars in the host galaxy.

Although it is difficult to quantify the significance of the difference between samples, it seems that optically obscured quasars (and optically unobscured but X-ray-absorbed quasars) have, on average, higher far-infrared luminosities than unobscured quasars. In turn, this implies that the cool-dust content of the host galaxies are different so that, on average, obscured quasars are hosted by dustier galaxies.

This is, of course, partly a result of selection. The samples selected to contain obscured sources will of course be biased toward dustier objects, but such a bias will only show in the farinfrared observations if obscuring dust on kpc scales is important (as opposed to dust in the torus only, which will emit mostly at mid-infrared wavelengths). The S05 sample is particularly biased toward such host obscuration, since it contains optically

\footnotetext{
15 It is difficult to quantify accurately the statistical significance of the differences between samples. As quoted in the ASURV manual: "It is possible that all existing survival methods will be inaccurate for astronomical data sets containing many points very close to the detection limit" (Isobe \& Feigelson 1990), which is clearly the case in this comparison. To make things worse, the individual samples considered here are small in a statistical sense: they all consist of less than 30 objects.
}

bright quasars that are not obscured by the torus, but which show absorption at X-ray energies.

Hence, the difference in far-infrared luminosities between obscured and unobscured quasars lends strong support to the scenario that some of the obscuring dust is in the host galaxy, not only the torus, and there is a hint that many obscured quasars might represent a different evolutionary phase to unobscured quasars.

\section{DISCUSSION AND CONCLUSIONS}

We have observed a sample of $z \gtrsim 2$ radio-intermediate obscured quasars at $1.2 \mathrm{~mm}(250 \mathrm{GHz})$ using MAMBO. The detection rate is five out of $21(24 \%)$. Sources with narrow lines seem to have a higher detection rate than sources with no narrow lines, but with such small numbers the difference is not significant. Larger samples will be required to study any differences.

Stacking leads to a statistical detection of $\left\langle S_{1.2} \mathrm{~mm}\right\rangle=0.96 \pm$ $0.11 \mathrm{mJy}$. Even if only the non-detections are stacked, they still yield a statistical detection, with $\left\langle S_{1.2} \mathrm{~mm}\right\rangle=0.51 \pm 0.13 \mathrm{mJy}$. Thus, the typical flux density of this sample is $\sim 0.5-1.0 \mathrm{mJy}$, and this corresponds to a far-infrared luminosity $\sim 4 \times 10^{12} L_{\odot}$.

If the far-infrared luminosity is powered entirely by star formation, and not by AGN-heated dust, then the typical inferred SFR is $\sim 700 M_{\odot} \mathrm{yr}^{-1}$. This large SFR is comparable to those inferred in ULIRGs and SMGs, the most powerful starburst galaxies known.

The observations at $1.2 \mathrm{~mm}$ also allow us to estimate the mass of the cool dust, and we find a typical mass of $\sim 3 \times 10^{8} M_{\odot}$. If heated by young stars, this dust is expected to be distributed on $\sim 2 \mathrm{kpc}$ scales (the scale found for SMGs). If it is heated by the central AGN, with $L_{\mathrm{bol}} \sim 10^{13} L_{\odot}$, then it is expected to be distributed on scales $\lesssim 10 \mathrm{kpc}$.

Indeed, we estimate that such a large mass of cool dust is capable of causing alone the large values of $A_{\mathrm{V}}$ in this sample, without help of an obscuring torus.

Combining our observations at mid-infrared and millimeter wavelengths, we present dust SEDs for our sample, and derive a typical SED for our sample of high-redshift obscured quasars. The clumpy torus models cannot reproduce the SED. However, clumpy torus models with an additional screen of cold dust (and the corresponding far-infrared emission) do reproduce this mean SED. The amount of extinction from this additional screen is derived from the cool-dust mass, from the observations at $1.2 \mathrm{~mm}$. The depth of the silicate feature can be consistently achieved by the inferred cool-dust mass provided the dust is on scales $\lesssim 2 \mathrm{kpc}$, which is in excellent agreement with the typical radius of the far-infrared emission in SMGs. Again, this lends support to kpc-scale dust along the host galaxy playing an important role in the obscuration of these sources.

Obscuration by dust on kpc scales would also explain why in about half of the sample the narrow emission lines from the central AGN are not detectable, as well as why in some cases the jets seem to be pointing toward us, something unexpected if they are only obscured by the torus of the unified schemes.

However, we remind the reader that unobscured quasars have also been found to have similar cool-dust masses, and that in our sample there is not a one-to-one correspondence between the presence (or lack of) narrow lines and detection at $1.2 \mathrm{~mm}$. The presence of a large mass of dust does not guarantee obscuration. A clumpy dusty interstellar medium where different lines of sight have vastly different optical depths is a likely explanation 
for why some quasars with large dust masses are obscured while others are not.

When comparing with other samples of high-redshift quasars, we find that the obscured quasars probably have a higher fraction of their luminosity emerging at far-infrared wavelengths, compared to unobscured quasars. The far-infrared emission is isotropic, so that this difference cannot be ascribed to orientation-dependent obscuration: obscured quasars are likely to have higher far-infrared luminosities and cool-dust masses than unobscured quasars, suggesting the host galaxies are in a dustier, presumably earlier, phase.

Additionally, we have searched for molecular gas in two sources at $z=2.767$ and 4.169, using the CO (3-2) and (43) transitions. In the $z=2.767$ source, we detect a line with $L_{\mathrm{CO}(3-2)}=3.2 \times 10^{7} L_{\odot}$ (equivalent to a brightness-temperature luminosity of $L_{\mathrm{CO}(3-2)}^{\prime}=2.4 \times 10^{10} \mathrm{~K} \mathrm{~km} \mathrm{~s}^{-1} \mathrm{pc}^{2}$ ). In the other source, the lack of detection suggests a line luminosity $L_{\mathrm{CO}(4-3)}<3 \times 10^{7} L_{\odot}\left(L_{\mathrm{CO}(4-3)}^{\prime}<1 \times 10^{10} \mathrm{~K} \mathrm{~km} \mathrm{~s}^{-1} \mathrm{pc}^{2}\right)$. Under the assumption that in these objects the (3-2) and (43 ) transitions are thermalized, we can estimate the molecular gas contents to be $M_{\mathrm{H}_{2}}=1.9 \times 10^{10}$ and $<8 \times 10^{9} M_{\odot}$, respectively. The estimated gas depletion timescales are $\tau_{\mathrm{g}}=4$ and $<16 \mathrm{Myr}$, and low gas-to-dust mass ratios of $M_{\mathrm{g}} / M_{\mathrm{d}}=19$ and $<20$ are inferred. A dynamical mass of $M_{\text {dyn }} \sin ^{2} i=6 \times$ $10^{9} M_{\odot}$ is estimated from the $\mathrm{CO}(3-2)$ detection.

We thank the IRAM staff for help with the observations and data reduction for this program. We are particularly grateful to Stéphane Leon for extensive help with the observations at the $30 \mathrm{~m}$, Robert Zylka for making the MOPSIC package publicly available, Jan Martin Winters for help with the PdBI observations, Philippe Salomé for help reducing the PdBI data on AMS16 and Roberto Neri for use of his software. We also thank Javier Rodón, Veronica Roccatagliata, and Aurora Sicilia-Aguilar for help with software. We thank the support and assistance provided by the CSO staff and SHARC-II team at Caltech during the observations and data reduction. We are also grateful to the CLUMPY group ${ }^{16}$ for making their models publicly available. This manuscript was improved by the suggestions of an anonymous referee. The work was partially supported by grants associated with Spitzer programs GO20705 and GO-30634, and is based on observations made with the Spitzer Space Telescope, which is operated by the Jet Propulsion Laboratory, California Institute of Technology under a contract with NASA.

\section{REFERENCES}

Alton, P. B., Xilouris, E. M., Misiriotis, A., Dasyra, K. M., \& Dumke, M. 2004, A\&A, 425, 109

Aravena, M., et al. 2008, A\&A, 491, 173

Armus, L., et al. 2007, ApJ, 656, 148

Barger, A. J., Cowie, L. L., Mushotzky, R. F., Yang, Y., Wang, W.-H., Steffen, A. T., \& Capak, P. 2005, AJ, 129, 578

Barger, A. J., Cowie, L. L., Sanders, D. B., Fulton, E., Taniguchi, Y., Sato, Y., Kawara, K., \& Okuda, H. 1998, Nature, 394, 248

Barvainis, R. 1987, ApJ, 320, 537

Beelen, A., Cox, P., Benford, D. J., Dowell, C. D., Kovács, A., Bertoldi, F., Omont, A., \& Carilli, C. L. 2006, ApJ, 642, 694

Beelen, A., et al. 2004, A\&A, 423, 441

Brand, K., et al. 2007, ApJ, 663, 204

Chapman, S. C., Smail, I., Windhorst, R., Muxlow, T., \& Ivison, R. J. 2004, ApJ, 611,732

Clavel, J., et al. 2000, A\&A, 357, 839

$\overline{16 \text { http://www.pa.uky.edu/clumpy/index.html }}$
Cole, S., et al. 2001, MNRAS, 326, 255

Coleman, G. D., Wu, C.-C., \& Weedman, D. W. 1980, ApJS, 43, 393

Croom, S. M., Smith, R. J., Boyle, B. J., Shanks, T., Miller, L., Outram, P. J., \& Loaring, N. S. 2004, MNRAS, 349, 1397

Dowell, C. D., et al. 2003, Proc. SPIE, 4855, 73

Downes, D., \& Solomon, P. M. 1998, ApJ, 507, 615

Dunne, L., Eales, S. A., \& Edmunds, M. G. 2003, MNRAS, 341, 589

Egami, E., et al. 2004, ApJS, 154, 130

Elvis, M., et al. 1994, ApJS, 95, 1

Fabian, A. C. 1999, MNRAS, 308, L39

Fadda, D., et al. 2006, AJ, 131, 2859

Frayer, D. T., et al. 2004, ApJS, 154, 137

Frayer, D. T., et al. 2006, AJ, 131, 250

Greve, T. R., et al. 2005, MNRAS, 359, 1165

Haas, M., et al. 2004, A\&A, 424, 531

Houck, J. R., et al. 2005, ApJ, 622, L105

Hughes, D. H., et al. 1998, Nature, 394, 241

Isobe, T., \& Feigelson, E. D. 1990, BAAS, 22, 917

Kennicutt, R. C., Jr. 1998, ARA\&A, 36, 189

Klamer, I. J., Ekers, R. D., Sadler, E. M., Weiss, A., Hunstead, R. W., \& De Breuck, C. 2005, ApJ, 621, L1

Klöckner, H., Martinez-Sansigre, A., Rawlings, S., \& Garrett, M. A. 2009, MNRAS, 398, 176

Kovács, A. 2006, PhD thesis, Caltech

Kovács, A., Chapman, S. C., Dowell, C. D., Blain, A. W., Ivison, R. J., Smail, I., \& Phillips, T. G. 2006, ApJ, 650, 592

Kreysa, E., et al. 1998, Proc. SPIE, 3357, 319

Lacy, M., Sajina, A., Petric, A. O., Seymour, N., Canalizo, G., Ridgway, S. E., Armus, L., \& Storrie-Lombardi, L. J. 2007, ApJ, 669, L61

Lacy, M., et al. 2005, ApJS, 161, 41

Lawrence, A., \& Elvis, M. 1982, ApJ, 256, 410

Leong, M., Peng, R., Houde, M., Yoshida, H., Chamberlin, R., \& Phillips, T. G. 2006, Proc. SPIE, 6275, 21

Levenson, N. A., Sirocky, M. M., Hao, L., Spoon, H. W. W., Marshall, J. A., Elitzur, M., \& Houck, J. R. 2007, ApJ, 654, L45

Lutz, D., Yan, L., Armus, L., Helou, G., Tacconi, L. J., Genzel, R., \& Baker, A. J. 2005, ApJ, 632, L13

Martínez-Sansigre, A., Lacy, M., Sajina, A., \& Rawlings, S. 2008, ApJ, 674, 676

Martínez-Sansigre, A., Rawlings, S., Garn, T., Green, D. A., Alexander, P., Klöckner, H.-R., \& Riley, J. M. 2006a, MNRAS, 373, L80

Martínez-Sansigre, A., Rawlings, S., Lacy, M., Fadda, D., Jarvis, M. J., Marleau, F. R., Simpson, C., \& Willott, C. J. 2006b, MNRAS, 370, 1479

Martínez-Sansigre, A., Rawlings, S., Lacy, M., Fadda, D., Marleau, F. R., Simpson, C., Willott, C. J., \& Jarvis, M. J. 2005, Nature, 436, 666

Martínez-Sansigre, A., et al. 2007, MNRAS, 379, L6

Nenkova, M., Sirocky, M. M., Ivezić, Ž., \& Elitzur, M. 2008a, ApJ, 685, 147

Nenkova, M., Sirocky, M. M., Nikutta, R., Ivezić, Ž., \& Elitzur, M. 2008b, ApJ, 685,160

Neri, R., et al. 2003, ApJ, 597, L113

Omont, A., Beelen, A., Bertoldi, F., Cox, P., Carilli, C. L., Priddey, R. S., McMahon, R. G., \& Isaak, K. G. 2003, A\&A, 398, 857

Page, M. J., Stevens, J. A., Ivison, R. J., \& Carrera, F. J. 2004, ApJ, 611, L85

Papadopoulos, P., Ivison, R., Carilli, C., \& Lewis, G. 2001, Nature, 409, 58

Pei, Y. C. 1992, ApJ, 395, 130

Polletta, M., Weedman, D., Hönig, S., Lonsdale, C. J., Smith, H. E., \& Houck, J. 2008, ApJ, 675, 960

Reyes, R., et al. 2008, AJ, 136, 2373

Riechers, D. A., et al. 2006, ApJ, 650, 604

Rigby, J. R., Rieke, G. H., Donley, J. L., Alonso-Herrero, A., \& Pérez-González, P. G. 2006, ApJ, 645, 115

Sajina, A., Yan, L., Lacy, M., \& Huynh, M. 2007, ApJ, 667, L17

Sajina, A., et al. 2008, ApJ, 683, 659

Salpeter, E. E. 1955, ApJ, 121, 161

Sanders, D. B., Phinney, E. S., Neugebauer, G., Soifer, B. T., \& Matthews, K. 1989, ApJ, 347, 29

Sanders, D. B., Soifer, B. T., Elias, J. H., Madore, B. F., Matthews, K., Neugebauer, G., \& Scoville, N. Z. 1988, ApJ, 325, 74

Schweitzer, M., et al. 2006, ApJ, 649, 79

Siebenmorgen, R., Freudling, W., Krügel, E., \& Haas, M. 2004, A\&A, 421, 129

Simpson, C., Rawlings, S., \& Lacy, M. 1999, MNRAS, 306, 828

Smith, D. J. B., Jarvis, M. J., Simpson, C., \& Martínez-Sansigre, A. 2009, MNRAS, 393, 309

Solomon, P. M., \& Vanden Bout, P. A. 2005, ARA\&A, 43, 677 
Spoon, H. W. W., Keane, J. V., Tielens, A. G. G. M., Lutz, D., Moorwood, A. F. M., \& Laurent, O. 2002, A\&A, 385, 1022

Spoon, H. W. W., Marshall, J. A., Houck, J. R., Elitzur, M., Hao, L., Armus, L., Brandl, B. R., \& Charmandaris, V. 2007, ApJ, 654, L49

Stevens, J. A., Page, M. J., Ivison, R. J., Carrera, F. J., Mittaz, J. P. D., Smail, I., \& McHardy, I. M. 2005, MNRAS, 360, 610

Stevens, J. A., et al. 2003, Nature, 425, 264

Szokoly, G. P., et al. 2004, ApJS, 155, 271
Tacconi, L. J., et al. 2006, ApJ, 640, 228

Urry, C. M., \& Padovani, P. 1995, PASP, 107, 803

Valiante, E., Lutz, D., Sturm, E., Genzel, R., Tacconi, L. J., Lehnert, M. D., \& Baker, A. J. 2007, ApJ, 660, 1060

Wilman, R. J., Fabian, A. C., Crawford, C. S., \& Cutri, R. M. 2003, MNRAS, 338, L19

Younger, J. D., et al. 2008, ApJ, 688, 59

Zakamska, N. L., et al. 2003, AJ, 126, 2125 